\title{
A Renormalization Group Analysis of the Kosterlitz-Thouless Phase
}

\section{J. Dimock ${ }^{1}$ and T. R. Hurd ${ }^{2, \star}$}

1 Department of Mathematics, SUNY at Buffalo, Buffalo, NY 14214, USA

2 Department of Mathematics, McMaster University, Hamilton, Ontario, L8S 4K1, Canada

Received July 5, 1990

Abstract. We consider a classical Coulomb gas with a short distance cutoff in two dimensions; equivalently a Sine-Gordon field theory. For low temperature $\beta^{-1}$ and low activity $z$ the gas is in a multipole phase, the Kosterlitz-Thouless phase. For $\beta>8 \pi$ and $z$ sufficiently small we give a complete renormalization group analysis for this phase and show that the flow of the effective measures is toward a free field (infrared asymptotic freedom). This should lead to control over the long distance behavior of the theory.

\section{Introduction}

A classical Coulomb gas in two dimensions with inverse temperature $\beta$ and activity $z$ is defined by the grand canonical partition function

$$
\sum_{n=0}^{\infty} z^{n} / n !\left[\sum_{q_{1}, \ldots, q_{n}} \int d x_{1} \cdots d x_{n} \exp \left(-\beta / 2 \sum_{i, j} q_{i} q_{j} v\left(x_{i}-x_{j}\right)\right)\right],
$$

where the sum is over charges $q_{i}= \pm 1$. The potential $v(x-y)$ is the inverse Laplacian $\left(\Delta^{-1}\right)(x, y)$ with a short distance cutoff (essential for $\beta$ large). An equivalent expression is as a Sine-Gordon field theory, namely

$$
\int \exp \left(-2 z \int \cos \varphi(x) d x\right) d \mu_{\beta v}(\varphi)
$$

where $\mu_{\beta v}$ is a massless Gaussian measure with covariance $\beta v$.

For a dilute gas $(z$ small $)$ there is a phase transition as the temperature is lowered. At high temperatures ( $\beta$ small) there is a plasma phase with Debye screening and exponential decay of correlations. This was rigorously established by Brydges and Federbush [BF] and Yang [Y]. At low temperatures ( $\beta$ large) there is a multipole phase characterized by a power law decay of correlations. This

\footnotetext{
* Research supported by the Natural Sciences and Engineering Research Council
} 


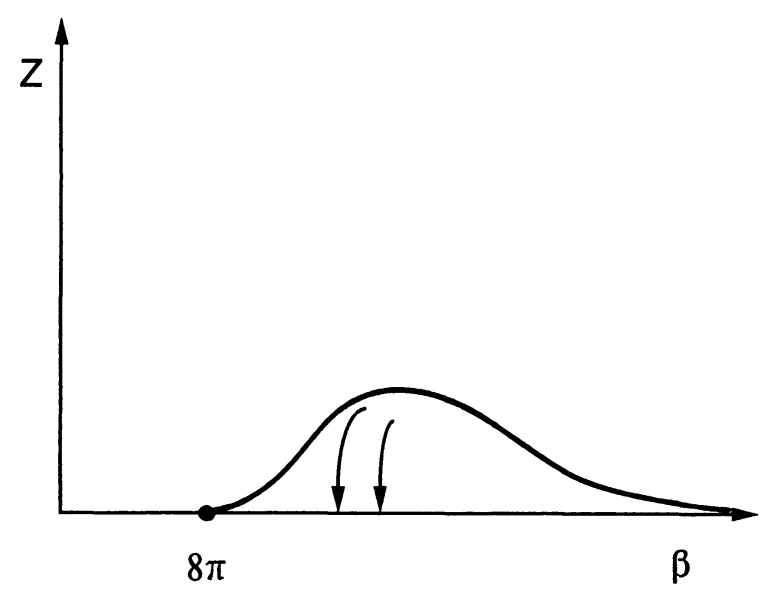

Fig. 1.

phase was predicted by Kosterlitz and Thouless [KT], and its existence was subsequently rigorously established by Fröhlich and Spencer [FS] (see also $[\mathrm{MKP}])$. Heuristic arguments suggest that the transition point is at $\beta=8 \pi$.

Our interest is in giving a complete renormalization group analysis of the Kosterlitz-Thouless phase. This involves successively integrating out short distance modes to leave effective measures for the long distance modes. One expects that the measures become increasingly Gaussian (i.e. like $\mu_{\beta v}$ ) as the iteration proceeds. One says that the theory is asymptotically free in the infrared.

Our main result is that for all $\beta>8 \pi$ and for $z$ sufficiently small (depending on $\beta$ ) we have infrared asymptotic freedom in the above sense. The region of validity is sketched in Fig. 1. We also sketch the flow of the renormalization group transformations as tracked by certain effective parameters $\beta, z$. It should follow from our results that the pressure exists in infinite volume and is analytic in $z$ near 0 . It should also follow that the infinite volume correlation functions exist and decay like those of the fixed point (i.e. as a power law).

An idealization of the Coulomb gas is the pure dipole gas. The Sine-Gordon transformation applied to this model leads to a field theory in which the interaction only depends on the derivatives $\partial \varphi$ of the field. This model has been previously analyzed by Gawedzki and Kupiainen [GK] using their powerful renormalization group techniques. They also treat the infinite volume pressure and correlation functions for the model.

Recently Brydges and Yau [BY] have provided an elegant and detailed framework for the dipole gas and related models which in several ways clarifies the earlier work of other authors. Among the improvements introduced in this paper are: (i) an identification of appropriate Banach spaces of effective interactions; (ii) no need to split into large and small field regions; (iii) a clear formulation of the polymer activity expansions scale by scale; (iv) a simplified treatment of the fluctuation integral at each scale.

In the present paper, we show that the methods of [BY] are also extremely useful for a field theory which depends on the scalar field $\varphi$. In two dimensions all local functions of the field $\varphi$ are superficially relevant variables, and this seems 
to work against asymptotic freedom. However, the Sine-Gordon theory has the extra feature that the effective interaction at each stage is a periodic function of $\varphi$ with period $2 \pi$ and can be analyzed by Fourier series. We carry this out by first reducing the $\varphi$ dependence to a dependence on $\{\varphi(x)\}$ for a finite number of points $x$ by a judicious use of the identity

$$
\varphi(\cdot)=\varphi(x)+\int_{x}^{(\cdot)} \partial_{\mu} \varphi d x^{\mu}
$$

Then we Fourier analyze in the variables $\{\varphi(x)\}$. The zero modes are then functions only of $\partial \varphi$ and local functions of $\partial \varphi$ are marginal or irrelevant and so can be treated as for the dipole gas. The non-zero modes are irrelevant because of a mechanism which corresponds physically to the suppression of charged particle distributions.

To illustrate this new mechanism, suppose there is only one degree of freedom. Then the Fourier modes are the functions $\exp (i \varphi q)$, the fluctuation integral is effected by convolution with a Gaussian measure $d \mu_{\beta}$ of covariance $\beta$, and we have

$$
\int \exp (i q(\varphi+\zeta)) d \mu_{\beta}(\zeta)=\exp \left(-1 / 2 \beta|q|^{2}\right) \exp (i q \varphi)
$$

which shows the contraction for $q \neq 0$. The above is exactly the situation for a hierarchical version of the Coulomb gas. (The hierarchical model was recently treated in this fashion by Dimock [D]. However this paper turns out to substantially duplicate earlier work of Marchetti and Perez [MP]. For other treatments of the hierarchical model see [BGN], [KPW].)

For the full model our task is to develop a systematic framework for treating the $\{\varphi(x)\}$ dependence and the $\partial \varphi$ dependence at the same time. Given this, we then want to show that it is exactly for $\beta>8 \pi$ that the contractive factors dominate other growth factors in the problem.

The methods of this paper are also useful for studying the infrared properties of QED in dimensions $d \geqq 2$, a problem we take up elsewhere [DH].

\section{Renormalization Group Transformations}

2.1. We define the initial measure in detail. The base space is taken to be the 2-torus with sides of length $L^{N}$ for integral $L, N$,

$$
\Lambda=\Lambda(N)=\mathbf{R}^{2} / L^{N} \mathbf{Z}^{2} .
$$

Define $v^{0}=(-\Delta)^{-1}$ on (const.) $)^{\perp}$ and $v^{0}=0$ on constants (the value of $v^{0}$ on constants should not matter in the infinite volume limit). Then $v^{0}$ has the kernel:

$$
v^{0}(x-y)=|\Lambda|^{-1} \sum_{p \in \Lambda^{*} \backslash\{0\}} e^{i p(x-y)}\left(p^{2} e^{t^{2} p^{4}}\right)^{-1}
$$

for $t=0$, where $\Lambda^{*}=2 \pi L^{-N} \mathbf{Z}^{2}$. For $t>0$ there is an ultraviolet cutoff: we take $t=1$ for simplicity, but our results hold for any $t>0$. Note that the kernel is $C^{\infty}$.

Let $\mu_{\beta v^{0}}$ be the Gaussian measure with covariance $\beta v^{0}$ on the restricted Sobolev space $\left\{\varphi \in \mathscr{H}_{s}(\Lambda): \int \varphi=0\right\}$. Here $s$ is a fixed integer and we assume $s>3$ so for $\varphi \in \mathscr{H}_{s}(\Lambda)$ the function and its derivatives $\partial^{\alpha} \varphi, 0 \leqq|\alpha| \leqq 2$, are bounded continuous functions of $\Lambda$. 
For $X \subset \Lambda$ define

$$
V_{X}(\varphi)=2 z \int_{X} \cos \varphi(x) d x
$$

for $z \in \mathbf{R}$. Then the model is defined by the measure

$$
Z^{0}(\varphi) d \mu_{\beta v^{0}}(\varphi)=\exp \left(-V_{\Lambda}(\varphi)\right) d \mu_{\beta v^{0}}(\varphi)
$$

for $\beta>0$.

We rewrite (2.4) by making a Mayer expansion:

$$
\begin{aligned}
Z^{0} & =\exp \left(-\sum_{\Delta} V_{\Delta}\right) \\
& =\sum_{\left\{\Delta_{i}\right\}} \prod_{i}\left(\exp \left(-V_{\Delta_{\mathfrak{i}}}\right)-1\right) \\
& =\sum_{\left\{X_{i}\right\}} \prod_{i} K^{0}\left(X_{i}\right) .
\end{aligned}
$$

Here, in the first step, we break up $V_{\Lambda}$ into a sum over unit blocks $\Delta \subset \Lambda$ centered on points in $\mathbf{Z}^{2}$. In the second step the sum is over collections of unit blocks $\left\{\Delta_{i}\right\}$. Finally in the last step the blocks are grouped together into connected sets. We use the convention that the blocks are closed so two blocks are connected if they only have an edge or a corner in common. The last sum is over all disjoint collections $\left\{X_{i}\right\}$, where $X_{i}$ is a union of unit blocks. The polymer activities of the initial interaction are thus given by:

$$
K^{0}(X, \varphi)= \begin{cases}\prod_{\Delta \subset X}\left(\exp \left(-V_{\Delta}(\varphi)\right)-1\right) & \text { if } X \text { connected } \\ 0 & \text { otherwise. }\end{cases}
$$

We further rewrite (2.5) as follows [BY]. For functions $X \rightarrow K(X)$ on unions of cells ("cell" = open blocks, open edge, or corner point) define the circle product

$$
\left(K_{1} \circ K_{2}\right)(X)=\sum_{\substack{Y \cup Z=X \\ Y \cap Z=\varnothing}} K_{1}(Y) K_{2}(Z)
$$

and the circle exponential

$$
\mathscr{E} \times \mathrm{xp} K=\mathscr{I}+K+1 / 2 K \circ K+\cdots,
$$

where $\mathscr{I}(X)=1$ if $X=\varnothing$ and $\mathscr{I}(X)=0$ otherwise. We also define $\square(X)=1$ if $X$ is a cell and $\square(X)=0$ otherwise.

Now enlarge the sum in (2.5) to a sum over partitions of $\Lambda$ into unions of cells, defining $K^{0}(X)=0$ if $X$ is not a union of closed blocks. Then we have

$$
\begin{aligned}
Z^{0} & =\sum_{\left\{X_{i}\right\}} \prod_{i}\left(\square+K^{0}\right)\left(X_{i}\right) \\
& =\sum_{n=0}^{\infty} 1 / n ! \sum_{\left(X_{1}, \ldots, X_{n}\right)} \prod_{i}\left(\square+K^{0}\right)\left(X_{i}\right) \\
& =\mathscr{E} \operatorname{xp}\left(\square+K^{0}\right) .
\end{aligned}
$$

2.2. We perform renormalization group transformations designed to isolate the long distance behavior. A single step consists of three parts: (i) a fluctuation integral: high frequency modes are integrated out, (ii) relevant terms are isolated and the 
theory is reblocked on a larger scale, and (iii) we return to the original scale, but in a smaller volume. The treatment follows that of Brydges and Yau [BY]. (However they use the order (ii), (iii), (i).)

After $j$ steps the base space is $\Lambda_{j}=\Lambda(N-j)$ and we have a measure

$$
Z^{j}(\varphi) d \mu_{\beta v}(\varphi)=\mathscr{E} \times p\left(\square+K^{j}(\varphi)\right) d \mu_{\beta v}(\varphi)
$$

on functions on $\Lambda_{j}$. The covariance of the Gaussian part is the operator $v^{j}$ with kernel

$$
v^{j}(x-y)=\left|\Lambda_{j}\right|^{-1} \sum_{p \in \Lambda_{j}^{*} \backslash\{0\}} e^{i p(x-y)}\left(p^{2} e^{p^{4}}+\sigma^{j} p^{2}\right)^{-1}
$$

for some constants $\sigma^{j}$.

The functionals $K^{j}(X, \varphi)$ are still defined for unions of unit blocks $X$, although now $X$ need not be connected. The dependence on $\varphi$ is to be localized near $X$ in a sense to be made precise. We assume that if $S$ is any symmetry of $\mathbf{Z}^{2}$ then $K^{j}(S X, S \varphi)=K^{j}(X, \varphi)$. We also make the periodicity assumption $K^{j}(X, \varphi+2 \pi)=$ $K^{j}(X, \varphi)$.

We isolate the dependence of $K^{j}(X, \varphi)$ on low order derivatives and require that there are functionals $K^{j}(X, \psi)$ defined on triples $\psi=\left(\psi_{0}, \psi_{1}, \psi_{2}\right)$ of continuous functions $\psi_{\alpha}: \Lambda^{j} \rightarrow \mathbf{R}^{2^{\alpha}}$ such that

$$
K^{j}(X, \varphi)=K^{j}\left(X, \psi_{\varphi}\right)
$$

where

$$
\psi_{\varphi}=\left(\varphi, \partial_{\mu} \varphi, \partial_{\mu} \partial_{v} \varphi\right)
$$

The $K^{j}(X, \psi)$ are to have the same support, symmetry, and periodicity properties as $K^{j}(X, \varphi)$. We also assume $K^{j}(X, \psi)$ is analytic in $\psi$ (see Sect. 3). Still there is some freedom in the choice of $K^{j}(X, \psi)$ which will be useful.

2.3. We now explain in detail the transformation from $j$ to $j+1$ starting with the fluctuation integral.

Define a new covariance $v^{\#}$ on $\Lambda_{j}$ by

$$
\tilde{v}^{\#}(p)= \begin{cases}\left(p^{2}\right)^{-1}\left(e^{L^{4} p^{4}}+\sigma^{j}\right)^{-1} & \text { if } p \neq 0 \\ 0 & \text { if } \quad p=0 .\end{cases}
$$

i.e. replace $e^{p^{4}}$ by $e^{L^{4} p^{4}}$ in (2.10). Then define the fluctuation covariance $C$ by $v^{j}=v^{\#}+C$ so that

$$
\tilde{C}(p)=p^{-2}\left[\left(e^{p^{4}}+\sigma^{j}\right)^{-1}-\left(e^{L^{4} p^{4}}+\sigma^{j}\right)^{-1}\right] .
$$

Note that this is smooth and vanishes at $p=0$ which is why we took a cutoff $e^{p^{4}}$ rather than say $e^{p^{2}}$.

Now integrals with respect to $d \mu_{\beta v^{j}}$ can be expressed as integrals with respect to $d \mu_{\beta v^{*}} \times d \mu_{\beta c}$. If we define

$$
Z^{\#}(\varphi)=\left(\mu_{\beta C} * Z^{j}\right)(\varphi) \equiv \int Z^{j}(\varphi+\zeta) d \mu_{\beta C}(\zeta)
$$

then $\int Z^{\#} d \mu_{\beta v^{\sharp}}=\int Z^{j} d \mu_{\beta v}$. The new density $Z^{\#}$ has a local expansion $Z^{\#}=$ $\mathscr{E} \mathrm{xp}\left(\square+K^{\#}\right)$, where $K^{\#}$ has the same properties as $K^{j}$. There is an explicit formula for $K^{\sharp}$ (see Sect. 6). 

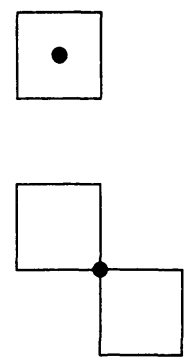

Fig. 2.
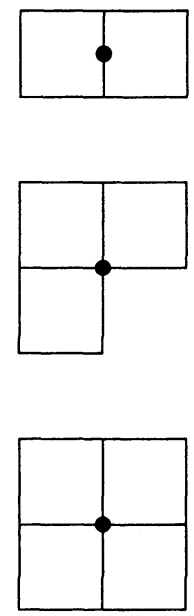

We next extract relevant and marginal pieces for $K^{\#}(X, \psi)$. The first step is to reduce the $\psi_{0}$ dependence to a single point. A set $X$ is said to be small $(X \in \mathscr{S})$ if the centers of the constituent blocks have diameter $\leqq 1$ in an $l^{\infty}$ metric. The possible small sets are shown in Fig. 2. For each small set $X$ we pick a distinguished point $x \in X$ as indicated in the figure. The choice is unaffected by lattice symmetries.

We define for $X \in \mathscr{S}, \Phi \in \mathbf{R}$, and $\hat{\psi}=\left(\psi_{1}, \psi_{2}\right)$,

$$
\left(K^{\#}\right)^{\prime}(X, \Phi, \hat{\psi})=K^{\#}\left(X, \Phi+\int_{x}^{(\cdot)} \psi_{1}, \hat{\psi}\right),
$$

where the integral is along a straight line. Then $\left(K^{\#}\right)^{\prime}$ is equivalent to $K^{\#}$ (written $\left.\left(K^{\#}\right)^{\prime} \doteq K^{\#}\right)$ in the sense that they agree when $\Phi=\varphi(x), \psi=\psi_{\varphi}$.

Now $\left(K^{\#}\right)^{\prime}$ is periodic in $\Phi$ and we may expand in a Fourier series. The Fourier coefficients for $q \in \mathbf{Z}$ are:

$$
k^{\#}(X, q, \hat{\psi})=(2 \pi)^{-1} \int_{-\pi}^{\pi} e^{i q \Phi}\left(K^{\#}\right)^{\prime}(X, \Phi, \widehat{\psi}) d \Phi .
$$

The relevant terms are from the low order terms in a Taylor expansion of $k^{\#}(X, q=0, \hat{\psi})$. These are (for $\left.X \in \mathscr{S}\right)$

$$
F(X, \hat{\psi})=\delta E^{j}(X)-\beta^{-1} / 2 \sum_{\mu, v} \int_{X} \psi_{1, \mu}(x) \delta \sigma_{\mu v}^{j}(X) \psi_{1, v}(x) d x,
$$

where

$$
\begin{aligned}
\delta E^{j}(X) & =k^{\#}(X, q=0, \hat{\psi}=0), \\
\delta \sigma_{\mu \nu}^{j}(X) & =-\beta /|X| \int\left[\frac{\delta k^{\#}(X, q=0)}{\delta \psi_{1, \mu}(x) \delta \psi_{1, \nu}(y)}\right](\hat{\psi}=0) d x d y
\end{aligned}
$$

(the notation is explained more fully in Sect. 3). We calculate

$$
\sum_{X} F(X, \hat{\psi})=\left|\Lambda_{j}\right| \delta E^{j}-1 / 2 \beta^{-1} \delta \sigma^{j} \int_{\Lambda}\left|\psi_{1}\right|^{2},
$$


where

$$
\begin{aligned}
\left|\Lambda_{j}\right| \delta E^{j} & =\sum_{X} \delta E^{j}(X), \\
\delta \sigma^{j} \delta_{\mu \nu} & =\sum_{X \supset \Delta} \delta \sigma_{\mu \nu}^{j}(X), \text { any } \Delta .
\end{aligned}
$$

Here we have used the lattice symmetry to obtain the $\delta_{\mu v}$.

We define $Z^{*}$ by extracting a Gaussian piece from $Z^{\#}$,

$$
Z^{\#}(\varphi)=\exp \left(\left|\Lambda_{j}\right| \delta E^{j}-1 / 2 \beta^{-1} \delta \sigma^{j} \int|\partial \varphi|^{2}\right) Z^{*}(\varphi) .
$$

Then $Z^{*}$ has a local expansion $Z^{*}=\mathscr{E} \times p\left(\square+K^{*}\right)$ and $K^{*}$ has the same properties as $K^{j}$, except that now $K^{*}=K^{*}(U, \psi)$ is only non-zero for sets $U$ which are unions of $L$-blocks. The explicit formula for $K^{*}$ is given in Sect. 7.

Next we absorb the quadratic piece into the measure:

$$
\exp \left(\left|\Lambda_{j}\right| \delta E^{j}-1 / 2 \beta^{-1} \delta \sigma^{j} \int|\partial \varphi|^{2}\right) d \mu_{\beta v^{\#}}=\mathscr{N}_{j} d \mu_{\beta v^{*}},
$$

where

$$
\begin{aligned}
& \tilde{v}^{*}(p)= \begin{cases}p^{-2}\left(e^{L^{4} p^{4}}+\sigma^{j}+\delta \sigma^{j}\right)^{-1} & \text { if } p \neq 0, \\
0 & \text { if } p=0\end{cases} \\
& \mathscr{N}_{j}=\exp \left(\left|\Lambda_{j}\right| \delta E^{j}\right) \int \exp \left(-1 / 2 \beta^{-1} \delta \sigma^{j} \int|\partial \varphi|^{2}\right) d \mu_{\beta v} \#(\varphi) .
\end{aligned}
$$

Then we have $\int Z^{\#} d \mu_{\beta v^{\#}}=\mathscr{N}_{j} \int Z^{*} d \mu_{\beta v^{*}}$.

Finally we scale down to $\Lambda_{j+1}$. With $\sigma^{j+1}=\sigma^{j}+\delta \sigma^{j}$ we have

$$
\tilde{v}^{j+1}(p)=L^{-2} \tilde{v}^{*}\left(L^{-1} p\right) \text {. }
$$

We also define

$$
K^{j+1}(X, \psi)=K^{*}(L X, R \psi),
$$

where $\left(R \psi_{\alpha}\right)(x)=L^{-\alpha} \psi_{\alpha}\left(L^{-1} x\right)$, and then $\int Z^{*} d \mu_{\beta v^{*}}=\int Z^{j+1} d \mu_{\beta v^{j-1}}$.

To summarize we have transformed the interaction density; $Z^{j} \rightarrow Z^{\#} \rightarrow Z^{*} \rightarrow Z^{j+1}$ in such a way that $\int Z^{j} d \mu_{\beta v^{j}}=\mathscr{N}_{j} \int Z^{j+1} d \mu_{\beta v^{j-1}}$. Our goal is to show that $Z^{j} \rightarrow 1$ as $j \rightarrow \infty$. At the same time we shall use the bounds we obtain for $\delta E^{j}$ and $\delta \sigma^{j}$ to control $\prod_{j} \mathscr{N}_{j}$.

\section{Analyticity and Norms}

We consider general functions $K(X, \psi)$ indexed by $X \in \Lambda$ and defined on triples of continuous functions $\psi=\left(\psi_{0}, \psi_{1}, \psi_{2}\right)$ on $\Lambda$. As explained in Sect. 2 , the polymer activities for our functional measures are restrictions of such functionals $K(X, \psi)$ to the subspace

$$
\mathscr{M}_{0}=\left\{\psi=\psi_{\varphi}: \varphi \in \mathscr{H}_{s}(\Lambda)\right\} .
$$

A basic regularity assumption is that $K(X, \psi)$ is an analytic functional on a neighbourhood of $\mathscr{M}_{0}$. That is, for any decomposition $\psi=\psi_{\varphi}+\mathbf{f}$ with $\psi_{\varphi} \in \mathscr{M}_{0}$ and $\mathbf{f}=\left(f_{0}, f_{1}, f_{2}\right)$ sufficiently small there is an absolutely convergent expansion

$$
K(X, \psi)=\sum_{\mathbf{n}} 1 / \mathbf{n} ! K_{\mathbf{n}}\left(X, \psi_{\varphi} ; \mathbf{f}^{\mathbf{n}}\right) .
$$


Here for $\mathbf{n}=\left(n_{0}, n_{1}, n_{2}\right)$ we have $\mathbf{n} !=n_{0} ! n_{1} ! n_{2} !$ and $\mathbf{f}^{n}=f_{0}^{n_{0}} f_{1}^{n_{1}} f_{2}^{n_{2}}$ (Cartesian product). The quantity $K_{\mathrm{n}}\left(X, \psi_{\varphi}\right)$ is a multilinear functional on $C(\Lambda, \mathbf{R})^{n_{0}} \times$ $C\left(\Lambda, \mathbf{R}^{2}\right)^{n_{1}} \times C\left(\Lambda, \mathbf{R}^{4}\right)^{n_{2}}$, symmetric on each sector and can be identified as the (Frechet) derivatives of $K(X, \psi)$ at $\psi=\psi_{\varphi}$.

The derivatives are assumed to have further regularity properties. Identify $C\left(\Lambda, \mathbf{R}^{2^{\alpha}}\right)$ with $C\left(\Lambda \times \Omega_{\alpha}\right)$, where $\Omega_{0}=\varnothing, \Omega_{1}=\{\mu\}, \Omega_{2}=\{\mu, v\}, 1 \leqq \mu, v \leqq 2$. We define $\hat{\Lambda}^{\mathrm{n}}=\left(\Lambda \times \Omega_{0}\right)^{n_{0}} \times\left(\Lambda \times \Omega_{1}\right)^{n_{1}} \times\left(\Lambda \times \Omega_{2}\right)^{n_{2}}$, and assume that $K_{\mathrm{n}}\left(X, \psi_{\varphi}\right)$ determines a continuous linear functional on $C\left(\hat{\Lambda}^{\mathrm{n}}\right)$ and thus a signed regular Borel measure on $\hat{\Lambda}^{\mathbf{n}}$. (Then $\mathbf{f}^{\mathrm{n}}$ is interpreted as the tensor product in $C\left(\hat{\Lambda}^{\mathrm{n}}\right)$.) For general $F \in C\left(\hat{\Lambda}^{\mathrm{n}}\right)$, we write formally

$$
K_{\mathrm{n}}\left(X, \psi_{\varphi} ; F\right)=\int\left[\frac{\delta^{N} K(X, \psi)}{\delta \psi\left(\xi_{1}\right) \cdots \delta \psi\left(\xi_{N}\right)}\right]_{\psi=\psi_{\varphi}} F\left(\xi_{1}, \ldots, \xi_{N}\right) d \xi_{1} \cdots d \xi_{N}
$$

where the integral is over $\hat{\Lambda}^{\mathbf{n}}$ and $N=|\mathbf{n}|$.

Norms on the functionals $K(X, \psi)$ are defined as follows. For the measure $K_{\mathrm{n}}\left(X, \psi_{\varphi}\right)$ we take the total variation norm, i.e. the norm as the dual of $C\left(\hat{\Lambda}^{\mathrm{n}}\right)$ :

$$
\left\|K_{\mathrm{n}}\left(X, \psi_{\varphi}\right)\right\|=\sup _{|F|_{\infty} \leqq 1}\left|K_{\mathrm{n}}\left(X, \psi_{\varphi} ; F\right)\right| .
$$

For any large field regulator

$$
G(X, \varphi)=\exp \left[\kappa / 2\|\varphi\|_{s, \tilde{X}}^{2}\right]
$$

$\left(\|\varphi\|_{s, \tilde{X}}\right.$ is a restricted Sobolev norm on $\tilde{X} \equiv X \cup\{$ corridor $\}$; see Eq. (5.2)), we define

$$
\left\|K_{\mathrm{n}}(X)\right\|_{G}=\sum_{\Delta} \sup _{\varphi \in \mathscr{H}_{s}}\left[\left\|K_{\mathrm{n}}\left(X, \psi_{\varphi}\right) 1_{\Delta}\right\| G(X, \varphi)^{-1}\right]
$$

where $\Delta=\Delta_{1} \times \cdots \times \Delta_{N}$ and each $\Delta_{i}$ is a closed unit square in $\Lambda$.

Next, for any large set regulator

$$
\Gamma(X)=A^{|X|} \Theta(X)
$$

(where $A=$ constant and $\Theta$ is an increasing function of the length of the shortest tree on $X$ ) we define

$$
\left\|K_{\mathrm{n}}\right\|_{G, \Gamma}=\sum_{X \ni \Delta_{0}} \Gamma(X)\left\|K_{\mathrm{n}}(X)\right\|_{G}
$$

Our functionals will be translation invariant so the norm does not depend on the choice of a fixed square $\Delta_{0}$.

Finally for $\mathbf{h}=\left(h_{0}, h_{1}, h_{2}\right), h_{\alpha} \geqq 0$, we define

$$
\|K\|_{G, \Gamma, \mathbf{h}}=\sum_{\mathbf{n}} \mathbf{h}^{\mathrm{n}} / \mathbf{n} !\left\|K_{\mathbf{n}}\right\|_{G, \Gamma} .
$$

Note that the $\mathbf{n}$ and $X$ summations can be interchanged:

where

$$
\|K\|_{G, \Gamma, \mathbf{h}}=\sum_{X \ni \Delta_{0}} \Gamma(X)\|K(X)\|_{G, \mathbf{h}}
$$

$$
\|K(X)\|_{G, \mathbf{h}}=\sum_{\mathbf{n}} \mathbf{h}^{\mathbf{n}} / \mathbf{n} !\left\|K_{\mathbf{n}}(X)\right\|_{G} .
$$


For each $\mathbf{h}$ let $\mathscr{M}_{\mathrm{h}}$ be the open neighborhood of $\mathscr{M}_{0}$ defined by

$$
\mathscr{M}_{\mathbf{h}}=\left\{\psi: \psi=\psi_{\varphi}+\mathbf{f}, \varphi \in \mathscr{H}_{s},\left|f_{\alpha}\right|_{\infty}<h_{\alpha}\right\} .
$$

Let $\mathscr{K}_{G, \Gamma, \mathbf{h}}$ be all functionals $K=K(X, \psi)$ defined for $\psi \in \mathscr{M}_{\mathbf{h}}$ with the following properties:

1. $K$ is analytic in the sense that it has expansions (3.2) with symmetric measure valued coefficients on each sector,

2. $\|K\|_{G, \Gamma, \mathbf{h}}<\infty$,

3. For any symmetry $S$ of $\mathbf{Z}^{2}, K(S X, S \psi)=K(X, \psi)$,

4. $K_{\mathrm{n}}\left(X, \psi_{\varphi}\right)$ is supported in $(\tilde{X})^{\mathrm{n}}$.

Note that for such a functional we have

$$
\left\|K_{\mathbf{n}}\left(X, \psi_{\varphi}\right)\right\| \leqq G(X, \varphi) \Gamma(X)^{-1} \mathbf{n} ! \mathbf{h}^{-\mathbf{n}}\|K\|_{G, \Gamma, \mathbf{h}} .
$$

It follows that the expansion (3.2) converges for all $\mathbf{f}=\left(f_{0}, f_{1}, f_{2}\right)$ with $\left|f_{\alpha}\right|_{\infty}<h_{\alpha}$ as required, and that

$$
|K(X, \psi)| \leqq G(X, \varphi) \Gamma(X)^{-1} \prod_{\alpha}\left(1-\left|f_{\alpha}\right|_{\infty} / h_{\alpha}\right)^{-1}\|K\|_{G, \Gamma, \mathrm{h}}
$$

The last inequality shows that $\|\cdot\|_{G, \Gamma, \mathbf{h}}$ is a norm on $\mathscr{K}_{G, \Gamma, \mathbf{h}}$.

Lemma 3.1. $\mathscr{K}_{G, \Gamma, \mathbf{h}}$ is a Banach space.

Proof. (Sketch) We must show it is complete. Let $K^{j}$ be a Cauchy sequence in $\mathscr{K}_{G, \Gamma, \mathbf{h}}$. By (3.12) we have that the limit $K(X, \psi)=\lim _{j \rightarrow \infty} K^{j}(X, \psi)$ exists for $\psi \in \mathscr{M}_{\mathbf{h}}$. By (3.11) $K_{n}^{j}\left(X, \psi_{\alpha}\right)$ is Cauchy sequence in the space of measures on $\hat{\Lambda}^{\mathbf{n}}$ and by the completeness of that space there are limiting measures $K_{\mathrm{n}}\left(X, \psi_{\alpha}\right)$. By limits one shows that $K(X, \psi)$ has the expansion (3.2) around any point $\psi_{\varphi}$ with coefficients $K_{\mathrm{n}}\left(X, \psi_{\varphi}\right)$. Now (1)-(4) follows, so $K \in \mathscr{K}_{G, \Gamma, \mathrm{h}}$. Finally one shows $\left\|K^{j}-K\right\|_{G, \Gamma, \mathbf{h}} \rightarrow 0$.

We say that $K$ is a local analytic functional if it is in $\mathscr{K}_{G, \Gamma, \mathbf{h}}$ for some $G, \Gamma, \mathbf{h}$. This definition only requires convergent expansions around points $\psi_{\varphi}$. But of course there are expansions around any $\psi \in \mathscr{M}_{\mathrm{h}}$. We have:

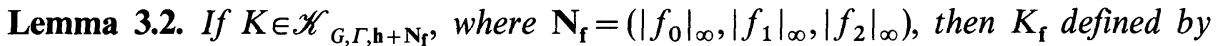
$K_{\mathrm{f}}(X, \psi)=K(X, \psi+\mathbf{f})$ is in $\mathscr{K}_{G, \Gamma, \mathbf{h}}$ and

$$
\left\|K_{\mathbf{f}}\right\|_{G, \Gamma, \mathbf{h}} \leqq\|K\|_{G, \Gamma, \mathbf{h}+\mathbf{N}_{\mathbf{f}}} .
$$

Proof. Let $\mathbf{g}$ satisfy $\left|g_{\alpha}\right|_{\infty}<h_{\alpha}$. Then $\psi+\mathbf{f}+\mathbf{g} \in \mathscr{M}_{\mathrm{h}+\mathbf{N}_{\mathrm{f}}}$ and

$$
\begin{aligned}
K_{\mathbf{f}}\left(X, \psi_{\varphi}+\mathbf{g}\right) & =K\left(X, \psi_{\varphi}+\mathbf{f}+\mathbf{g}\right) \\
& =\sum_{\mathbf{n}} 1 / \mathbf{n} ! K_{\mathbf{n}}\left(X, \psi_{\varphi} ;(\mathbf{f}+\mathbf{g})^{\mathbf{n}}\right) \\
& =\sum_{\mathbf{m}} 1 / \mathbf{m} !\left(K_{\mathbf{f}}\right)_{\mathbf{m}}\left(X, \psi_{\varphi} ; \mathbf{g}^{\mathbf{m}}\right),
\end{aligned}
$$

where

$$
\left(K_{\mathbf{f}}\right)_{\mathbf{m}}\left(X, \psi_{\varphi} ; \mathbf{g}^{\mathbf{m}}\right)=\sum_{\mathbf{n} \geqq \mathbf{m}} 1 /(\mathbf{n}-\mathbf{m}) ! K_{\mathbf{n}}\left(X, \psi_{\varphi} ; \mathbf{f}^{\mathbf{n}-\mathbf{m}} \mathbf{g}^{\mathbf{m}}\right)
$$


and $\mathbf{f}^{\mathbf{a}} \mathbf{g}^{\mathbf{b}}=f_{0}^{a_{0}} g_{0}^{b_{0}} f_{1}^{a_{1}} g_{1}^{b_{1}} f_{2}^{a_{2}} g_{2}^{b_{2}}$. The same formula defines a measure on $\hat{\Lambda}_{\mathbf{n}}$ and we have

$$
\left\|\left(K_{\mathbf{f}}\right)_{\mathbf{m}}\left(X, \psi_{\varphi}\right) 1_{\Delta}\right\| \leqq \sum_{\Delta^{\prime}} \sum_{\mathbf{n} \geqq \mathbf{m}} 1 /(\mathbf{n}-\mathbf{m}) !\left\|K_{\mathbf{n}}\left(X, \psi_{\varphi}\right) 1_{\Delta} 1_{\Delta^{\prime}}\right\| \mathbf{N}_{\mathbf{f}}^{\mathbf{n}-\mathbf{m}}
$$

and hence

$$
\begin{aligned}
\left\|K_{\mathbf{f}}\right\|_{G, \Gamma, \mathbf{h}} & \leqq \sum_{\mathbf{m}} \mathbf{h}^{\mathbf{m}} / \mathbf{m} !\left(\sum_{\mathbf{n} \geqq \mathbf{m}} \mathbf{N}_{\mathbf{f}}^{\mathbf{n}-\mathbf{m}} /(\mathbf{n}-\mathbf{m}) !\left\|K_{\mathbf{n}}\right\|_{G, \Gamma}\right) \\
& =\sum_{\mathbf{n}}\left(\mathbf{h}+\mathbf{N}_{\mathbf{f}}\right)^{\mathbf{n}} / \mathbf{n} !\left\|K_{\mathbf{n}}\right\|_{G, \Gamma} \\
& =\|K\|_{G, \Gamma, \mathbf{h}+\mathbf{N}_{\mathbf{f}}} \cdot \square
\end{aligned}
$$
define

The product of local analytic functionals is now considered. For $K \in \mathscr{K}_{G, \Gamma, \mathbf{h}}$ we

$$
|K|_{G, \Gamma, \mathrm{h}}=\sup _{X} \Gamma(X)\|K(X)\|_{G, \mathrm{~h}}
$$

and note that

$$
|K|_{G, \Gamma, \mathbf{h}} \leqq\|K\|_{G, \Gamma, \mathbf{h}} .
$$

Lemma 3.3. Let $K_{1} \in \mathscr{K}_{G_{1}, \Gamma_{1}, \mathrm{~h}}$ and $K_{2} \in \mathscr{K}_{G_{2}, \Gamma_{2}, \mathrm{~h}}$. Then $K_{1} K_{2} \in \mathscr{K}_{G_{1} G_{2}, \Gamma_{1} \Gamma_{2}, \mathrm{~h}}$ and (i) for all $X \in \Lambda$,

(ii)

$$
\left\|K_{1}(X) K_{2}(X)\right\|_{G_{1} G_{2}, \mathbf{h}} \leqq\left\|K_{1}(X)\right\|_{G_{1}, \mathbf{h}}\left\|K_{2}(X)\right\|_{G_{2}, \mathbf{h}}
$$

$$
\left\|K_{1} K_{2}\right\|_{G_{1} G_{2}, \Gamma_{1} \Gamma_{2}, \mathbf{h}} \leqq\left|K_{1}\right|_{G_{1}, \Gamma_{1}, \mathbf{h}}\left\|K_{2}\right\|_{G_{2}, \Gamma_{2}, \mathbf{h}} .
$$

Proof. Part (ii) is immediate from part (i), so we prove part (i). By definition, $\left(K_{1} K_{2}\right)(X, \psi)=K_{1}(X, \psi) K_{2}(X, \psi)$. We compute

$$
\left(K_{1} K_{2}\right)_{\mathbf{n}}\left(X, \psi_{\varphi}\right)=\sum_{\mathbf{a}+\mathbf{b}=\mathbf{n}} \mathbf{n} ! / \mathbf{a} ! \mathbf{b} ! \operatorname{Sym}\left[K_{1, \mathbf{a}}\left(X, \psi_{\varphi}\right) \otimes K_{2, \mathbf{b}}\left(X, \psi_{\varphi}\right)\right],
$$

where Sym denotes an average over permutations of the points for each sector. We find

$$
\begin{aligned}
&\left\|\left(K_{1} K_{2}\right)_{\mathbf{n}}(X)\right\|_{G_{1} G_{2}} \leqq \sum_{\mathbf{a}+\mathbf{b}=\mathbf{n}} \mathbf{n} ! / \mathbf{a} ! \mathbf{b} ! \sum_{\Delta, \Delta^{\prime}} \sup _{\varphi}\left[\left\|K_{1, \mathbf{a}}\left(X, \psi_{\varphi}\right) 1_{\Delta}\right\| G_{1}^{-1}\left(X, \psi_{\varphi}\right)\right] \\
& \cdot\left[\left\|K_{2, \mathbf{b}}\left(X, \psi_{\varphi}\right) 1_{\Delta^{\prime}}\right\| G_{2}^{-1}\left(X, \psi_{\varphi}\right)\right] \\
& \leqq \\
& \sum_{\mathbf{a}+\mathbf{b}=\mathbf{n}} \mathbf{n} ! / \mathbf{a} ! \mathbf{b} !\left\|K_{1, \mathbf{a}}(X)\right\|_{G_{1}}\left\|K_{2, \mathbf{b}}(X)\right\|_{G_{2}}
\end{aligned}
$$

and part (i) follows.

We now use these norms to bound the initial interaction given by the activity expansion with activities $K^{0}$ defined by (2.6).

Proposition 3.4. Let the large set regulator $\Gamma(X)=A^{\mid{ }^{x}} \mid \Theta(X)$ be such that $\Theta(X)=1$ if $X$ is a connected set. Then, for any $h_{0}>0$ with $48 A|z| e^{h_{0}+1}<1$, the functional $K^{0}$ is in $\mathscr{K}_{1, \Gamma, \mathbf{h}}$ and $\left\|K^{0}\right\|_{1, \Gamma, \mathbf{h}} \leqq \delta^{0} \equiv 6|z| A e^{h_{0}}$.

Proof. The functional $K^{0}$ depends only on $\psi_{0}=\varphi$, and we may ignore any reference 
to $\psi_{1}, \psi_{2}$. Clearly $V(\Delta, \varphi)$ is analytic and has derivatives

$$
V_{\mathrm{n}}(\Delta, \varphi ; F)=2 z \int_{\Delta} \cos ^{(n)}(\varphi(x)) F(x, \ldots, x) d x .
$$

Therefore $\left\|V_{\mathbf{n}}(\Delta, \varphi)\right\| \leqq 2|z|$ and so $V(\Delta) \in \mathscr{K}_{1, \mathbf{h}}$ with norm $\|V(\Delta)\|_{1, \mathbf{h}} \leqq 2|z| e^{h_{0}}$.

Next consider

$$
\exp (-V(\Delta, \varphi))-1=\sum_{n=1}^{\infty} V(\Delta, \varphi)^{N} / N !
$$

By Lemma 3.3(i)

$$
\left\|V(\Delta)^{N}\right\|_{1, \mathbf{h}} \leqq\|V(\Delta)\|_{1, \mathbf{h}}^{N}
$$

and so the series is norm convergent:

$$
\|\exp (-V(\Delta))-1\|_{1, \mathrm{~h}} \leqq \exp \left[2|z| e^{h_{0}}\right]-1 \leqq 3|z| e^{h_{0}} .
$$

For any connected set $X$, Lemma 3.3(i) implies

$$
\left\|K^{0}(X)\right\|_{1, \mathbf{h}} \leqq\left\|\prod_{\Delta \subset X}\left(e^{-V(\Delta)}-1\right)\right\|_{1, \mathbf{h}} \leqq\left(3|z| e^{h_{0}}\right)^{|X|},
$$

and thus

$$
\begin{aligned}
\left\|K^{0}\right\|_{1, \Gamma, \mathbf{h}} & =\sum_{X \ni \Delta_{0}} \Gamma(X)\left\|K^{0}(X)\right\|_{1, \mathbf{h}} \\
& \leqq \sum_{\substack{X \ni \Delta_{0} \\
X \text { connected }}}\left(3 A|z| e^{h}\right)^{|X|} .
\end{aligned}
$$

This standard sum is estimated by a spanning tree argument:

$$
\left.\sum_{\substack{X \ni \Delta_{0} \\ X \text { connected }}} r^{|X|} \leqq \sum_{N \geqq 1} r^{N} / N ! \sum_{\tau \text { on }\{1, \ldots, N\}} \#\left\{X \text { connected, } \ni \Delta_{0},|X|=N, \tau \subset G(X)\right)\right\},
$$

where $\tau$ is any spanning tree on $\{1, \ldots, N\}$ and we count sets $X$ whose connectivity graph $G(X)$ contains $\tau$. For each $\tau$, the number of such sets is $\leqq N \cdot 8^{N-1}$, Cayley's theorem bounds the number of trees by $N^{N-2}$, and so

$$
\left\|K^{0}\right\|_{1, \Gamma, \mathbf{h}} \leqq \sum_{N \geqq 1} r^{N} \cdot 8^{N-1} \cdot\left(N^{N-1} / N !\right)
$$

where $r=3 A|z| e^{h_{0}}$. By Stirling's formula, this is convergent provided $8 e r<1$, and for $8 e r<1 / 2$ the sum is bounded by $\delta^{0}=2 r$ (i.e. twice the $N=1$ term).

\section{Some Basic Lemmas}

In this section we want to establish bounds on $K^{\prime}, k$ (defined as in Eqs. (2.16) and (2.17)) in terms of $K$, and also bounds going the other way. We write $K^{\prime}$ as

$$
K^{\prime}(X, \Phi, \widehat{\psi})=K\left(X, \Phi+H \psi_{1}, \hat{\psi}\right), \quad X \in \mathscr{S},
$$

where $H=H(X)$ denotes the operator

$$
\left(H \psi_{1}\right)(y)=\int_{x}^{y} \psi_{1, \mu}(z) d z^{\mu}
$$


and $x \in X$ is the distinguished point. The Fourier coefficients are

$$
k(X, q, \hat{\psi})=(2 \pi)^{-1} \int_{-\pi}^{\pi} e^{-i q \Phi} K^{\prime}(X, \Phi, \hat{\psi}) d \Phi, \quad q \in \mathbf{Z} .
$$

We only consider functionals $K^{\prime}(X, \Phi, \hat{\psi})$ on small sets $X$. We define norms $\left\|K^{\prime} 1_{X \in \mathscr{S}}\right\|_{G, \Gamma, \mathbf{h}}$ just as in Sect. 3 except that now the first variable is in $\mathbf{C} \cong C(\{x\})$ instead of $C(\Lambda)$. Also, for the functionals $k(X, q, \hat{\psi})$ we have for each $q \in \mathbf{Z}$ a norm $\left\|k(q) 1_{X \in \mathscr{S}}\right\|_{G, \Gamma, \hat{\mathbf{h}}}$ which is just the restriction of $\|\cdot\|_{G, \Gamma, \mathbf{h}}$ to a functional independent of the 0 -component of $\psi$ (note $\hat{\mathbf{h}} \equiv\left(h_{1}, h_{2}\right)$ ).

Lemma 4.1. There is a constant $B$ so

(i)

(ii)

$$
\left\|K^{\prime} 1_{X \in \mathscr{S}}\right\|_{G, \Gamma, \mathbf{h}} \leqq\|K\|_{G, \Gamma, h_{0}+B h_{1}, \hat{\mathbf{h}}}
$$

$$
\left\|k(q) 1_{X \in \mathscr{P}}\right\|_{G, \Gamma, \hat{\mathbf{h}}} \leqq \exp \left[-h_{0}|q|\right]\left\|K^{\prime} 1_{X \in \mathscr{S}}\right\|_{G, \Gamma, \mathbf{h}} .
$$

Note: Combining (i) and (ii) with $h_{0} \rightarrow h_{0}-B h_{1}$ leads to

$$
\left\|k(q) 1_{X \in \mathscr{S}}\right\|_{G, \Gamma, \hat{\mathbf{h}}} \leqq \exp \left[-\left(h_{0}-B h_{1}\right)|q|\right]\|K\|_{G, \Gamma, \mathbf{h}} .
$$

Proof. (i) For $\mathbf{g}=\left(g_{0}, g_{1}, g_{2}\right) \in C(\{x\}) \times C\left(\Lambda \times \Omega_{1}\right) \times C\left(\Lambda \times \Omega_{2}\right)$

$$
\begin{aligned}
K^{\prime}\left(X, \Phi+g_{0}, \hat{\psi}_{\varphi}+\hat{\mathbf{g}}\right) & =K\left(X, \psi_{\varphi}+\left(g_{0}+H g_{1}, g_{1}, g_{2}\right)\right) \\
& =\sum_{\mathbf{n}} 1 / \mathbf{n} ! K_{\mathbf{n}}\left(X, \psi_{\varphi} ;\left(g_{0}+H g_{1}\right)^{n_{0}} g_{1}^{n_{1}} g_{2}^{n_{2}}\right) \\
& =\sum_{\mathbf{n}, l} 1 / \mathbf{n} !\left[\begin{array}{c}
n_{0} \\
l
\end{array}\right] K_{\mathbf{n}}\left(X, \psi_{\varphi} ; g_{0}^{n_{0}-l}\left(H g_{i}\right)^{l} g_{1}^{n_{1}} g_{2}^{n_{2}}\right) \\
& =\sum_{\mathbf{m}} 1 / \mathbf{m} ! K_{\mathbf{m}}^{\prime}\left(X, \Phi, \hat{\psi}_{\varphi} ; \mathbf{g}^{\mathbf{m}}\right) .
\end{aligned}
$$

In the last step we have made the change of variable $m_{0}=n_{0}-l, m_{1}=n_{1}+l$, $m_{2}=n_{2}$, and identified the derivatives of $K^{\prime}$ as

$$
K_{\mathrm{m}}^{\prime}\left(X, \Phi, \hat{\psi}_{\varphi} ; \mathbf{g}^{\mathrm{m}}\right)=\sum_{l}\left[\begin{array}{c}
m_{1} \\
l
\end{array}\right] K_{m_{0}+l, m_{1}-l, m_{2}}\left(X, \psi_{\varphi} ; g_{0}^{m_{0}}\left(H g_{1}\right)^{l} g_{1}^{m_{1}-l} g_{2}^{m_{2}}\right)
$$

A similar formula (with a symmetrization) defines a measure on $\hat{\Lambda}^{\mathrm{m}}$, and

$$
\begin{aligned}
\left\|K_{\mathbf{m}}^{\prime}\left(X, \Phi, \hat{\psi}_{\varphi}\right) 1_{\Delta}\right\| & \leqq \sum_{l}\left[\begin{array}{c}
m_{1} \\
l
\end{array}\right] \sum_{\Delta^{\prime}} \sup _{F \in C\left(\hat{A}^{\left.m^{m}\right):|F|_{\infty} \leqq 1}\right.} \mid K_{m_{0}+l, m_{1}-l, m_{2}}\left(X, \psi_{\varphi} ; 1_{\Delta^{\prime}} H^{l}\left(F 1_{\Delta}\right) \mid\right. \\
& \leqq \sum_{l}\left[\begin{array}{c}
m_{1} \\
l
\end{array}\right] \sum_{\Delta^{\prime}}\left\|1_{\Delta^{\prime}} H^{l}\left(\cdot 1_{\Delta}\right)\right\|\left\|K_{m_{0}+l, m_{1}-l, m_{2}}\left(X, \psi_{\varphi}\right) 1_{\Delta^{\prime}}\right\|,
\end{aligned}
$$

where the linear operator $F \rightarrow 1_{\Delta^{\prime}} H^{l}\left(F 1_{\Delta}\right)$ is bounded by (diameter $\left.(\Delta)\right)^{l}=(\sqrt{2})^{l}$. When we sup the bound over $\varphi$, the sum over $\Delta$ is done by noting the conditions

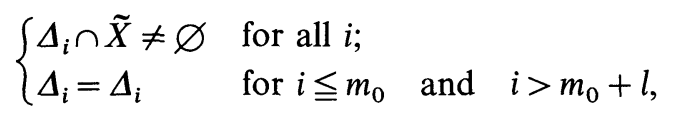

and hence

$$
\sum_{\Delta}\left\|1_{\Delta^{\prime}} H^{l}\left(\cdot 1_{\Delta}\right)\right\| \leqq B^{l}
$$


where $B=16 \sqrt{2}$. Now we have

$$
\begin{aligned}
\left\|K^{\prime} 1_{X \in \mathscr{S}}\right\|_{G, \Gamma, \mathbf{h}} & \leqq \sum_{\mathbf{m}, l} \mathbf{h}^{\mathbf{m}} / \mathbf{m} !\left[\begin{array}{c}
m_{1} \\
l
\end{array}\right] B^{l}\left\|K_{m_{0}+l, m_{1}-l, m_{2}}\right\|_{G, \Gamma} \\
& \leqq \sum_{\mathbf{n}, l} 1 / \mathbf{n} ! h_{0}^{n_{0}-l} h_{1}^{n_{1}} h_{2}^{n_{2}}\left(B h_{1}\right)^{l}\left[\begin{array}{c}
n_{0} \\
l
\end{array}\right]\left\|K_{\mathbf{n}}\right\|_{G, \Gamma} \\
& \leqq \sum_{\mathbf{n}} 1 / \mathbf{n} !\left(h_{0}+B h_{1}\right)^{n_{0}} h_{1}^{n_{1}} h_{2}^{n_{2}}\left\|K_{\mathbf{n}}\right\|_{G, \Gamma} \\
& =\|K\|_{G, \Gamma, h_{0}+B h_{1}, \hat{\mathbf{h}}} .
\end{aligned}
$$

(ii) If $K^{\prime} \in \mathscr{K}_{G, \Gamma \mathrm{h}}$, the functional $K^{\prime}$ is analytic in $\Phi$ on the strip $|\operatorname{Im} \Phi|<h_{0}$. In the formula for $k(q)$, with $q \neq 0$, we shift the integration contour by $\Phi \rightarrow \Phi \mp i\left(h_{0}-\varepsilon\right)$ ( \pm corresponding to $\pm q>0$; any $\varepsilon \in\left(0, h_{0}\right)$ ). Then

$$
k(X, q, \hat{\psi})=e^{-\left(h_{0}-\varepsilon\right)|q|}(2 \pi)^{-1} \int_{-\pi}^{\pi} e^{-i q \Phi} K^{\prime}\left(X, \Phi \mp i\left(h_{0}-\varepsilon\right), \hat{\psi}\right) d \Phi .
$$

The derivatives $k_{\hat{\mathbf{n}}}(q)$ are related to the derivatives $K_{0, \hat{\mathbf{n}}}^{\prime}$ by the same formula. This leads to

$$
\begin{aligned}
\left\|k(q) 1_{X \in \mathscr{S}}\right\|_{G, \Gamma, \hat{\mathbf{h}}} & \leqq e^{-\left(h_{0}-\varepsilon\right)|q|}\left\|K^{\prime}\left(\cdot \mp i\left(h_{0}-\varepsilon\right), \cdot, \cdot\right) 1_{X \in \mathscr{S}}\right\|_{G, \Gamma, 0, \hat{\mathbf{h}}} \\
& \leqq e^{-\left(h_{0}-\varepsilon\right)|q|}\left\|K^{\prime} 1_{X \in \mathscr{S}}\right\|_{G, \Gamma, h_{0}-\varepsilon, \hat{\mathbf{h}}}
\end{aligned}
$$

by Lemma 3.2. This bound, true for any $\varepsilon>0$, is therefore true for $\varepsilon=0$. The bound for $k(q=0)$ follows without shifting the contour.

If we start with $k$, we can recover $K^{\prime}$ by summing the Fourier series. If we then define $K^{\prime \prime}$ by $K^{\prime \prime}(X, \psi)=K^{\prime}\left(X, \psi_{0}(x), \hat{\psi}\right)$ then $K^{\prime \prime} \doteq K$, i.e. they agree on $\mathscr{M}_{0}$. In terms of $k$ we have

$$
K^{\prime \prime}(X, \psi)=\sum_{q \in \mathbf{Z}} e^{i q \psi_{0}(x)} k(X, q, \hat{\psi} \cdot)
$$

\section{Lemma 4.2.}

(i)

$$
\left\|e^{i q \psi(x)}\right\|_{G=1, h_{0}} \leqq e^{|q| h_{0}},
$$

(ii) If $\left\|k(q) 1_{X \in \mathscr{I}}\right\|_{G, \Gamma, \hat{\mathbf{h}}} \leqq C e^{-|q| h_{0}}$ then for any $\varepsilon>0$ there is a constant $C_{\varepsilon}$ so

$$
\left\|K^{\prime \prime} 1_{X \in \mathscr{S}}\right\|_{G, \Gamma, h_{0}-\varepsilon, \hat{\mathbf{h}}} \leqq C C_{\varepsilon} .
$$

Proof. (i) It is straightforward that $f(q, \psi)=e^{i q \psi_{0}(x)}$ is analytic and has derivatives

$$
f_{n_{0}}\left(q, \psi_{\varphi} ; F\right)=(i q)^{n_{0}} F(x, \ldots, x) e^{i q \varphi(x)} .
$$

We therefore have a bound $\left\|f_{n_{0}}(q)\right\|_{G=1} \leqq|q|^{n_{0}}$ and part (i) follows.

(ii) Now let $f(X, q, \psi)=e^{i q \psi_{0}(x)}$, where $x$ is the distinguished point in $X$. By Lemma 3.3 and (i):

$$
\begin{aligned}
\left\|K^{\prime \prime} 1_{X \in \mathscr{S}}\right\|_{G, \Gamma, h_{0}-\varepsilon, \hat{\mathbf{h}}} & \leqq \sum_{q}|f(q)|_{G=1, \Gamma=1, h_{0}-\varepsilon}\left\|k(q) 1_{X \in \mathscr{S}}\right\|_{G, \Gamma, \hat{\mathbf{h}}} \\
& \leqq C \sum_{q} e^{-|q| \varepsilon}=C \cdot C_{\varepsilon} \cdot \square
\end{aligned}
$$




\section{The Main Theorem}

The renormalization group transformations have given us a sequence $K^{0}, K^{1}, K^{2}, \ldots$ of interaction functionals. We want to show $K^{j} \rightarrow 0$ as $j \rightarrow \infty$, which we characterize in terms of the norms $\|\cdot\|_{G, \Gamma, \mathbf{h}}$. We discuss the specific choices of norms, state the result, and outline the proof.

It turns out that the large field and analyticity properties of the functionals deteriorate slightly as we iterate. No single norm is adequate and we must allow some weak dependence on $j$. Thus we consider $\left\|K^{j}\right\|_{G^{j}, \Gamma^{i} \mathbf{h}^{j}}$ with $G^{j+1}>G^{j}, G^{j} \cong G^{0}$ and $\mathbf{h}^{j+1}<\mathbf{h}^{j}, \mathbf{h}^{j} \cong \mathbf{h}^{0}$ and $\Gamma^{j}=\Gamma^{0}$. We want to show $\left\|K^{j}\right\|_{G^{j} \Gamma^{j}, \mathbf{h}^{j}}$ is decreasing in $j$.

A fundamental condition is that $\left\|K^{0}\right\|_{G^{0}, \Gamma^{0} \mathbf{h}^{0}}$ is sufficiently small. Since $\left\|K^{0}\right\|_{G^{0}, \Gamma^{0}, h^{0}} \leqq\left\|K^{0}\right\|_{1, \Gamma^{0}, h^{0}}$ it follows by Lemma 3.4 that this is true if $z$ is sufficiently small. However to minimize the constraint on $z$ we will not want to take $\Gamma, \mathbf{h}^{\mathbf{0}}$ any larger than necessary.

A second fundamental condition is that $L$ be sufficiently large. This guarantees a sufficiently strong contraction. In fact we will be able to show that

$$
\left\|K^{j+1}\right\|_{G^{j+1}, \Gamma^{j+1}, \mathbf{h}^{j+1}} \leqq L^{-\varepsilon}\left\|K^{j}\right\|_{G^{j}, \Gamma^{j}, \mathbf{h}^{j}}
$$

for any $\varepsilon<\min (1 / \beta / 4 \pi-2)$; for definiteness say $\varepsilon=\min (1 / 2, \beta / 8 \pi-1)$. (By comparison, for the dipole gas Brydges and Yau are able to obtain (4.1) with any $\varepsilon<1$, independent of $\beta$. Also, for their model analyticity properties improve and one can allow $h^{j}$ to grow in $j$. This improvement corresponds to the absence of marginal variables in the model.)

We now define $G^{j}, \Gamma^{j}, \mathbf{h}^{j}$. For the large field regulator we take:

$$
G^{j}(X, \varphi)=\exp \left(1 / 2 \kappa^{j} \sum_{0<|\alpha| \leqq s}\left\|\partial^{\alpha} \varphi\right\|_{X_{1 / 6}}^{2}\right) .
$$

Here $X_{\eta}$ is an enlargement of $X$ which has a smooth boundary and contains all points $y$ with $d(y, X) \leqq 3 \eta / 4$ but no points with $d(y, X)>\eta$. The norm $\|\cdot\|_{X}$ is the norm on $L^{2}(X)$. The exponent has a form suitable for dominating $\left|\partial^{\alpha} \varphi\right|$ by Sobolev inequalities for $|\alpha|=1,2$. The coefficient $\kappa^{j}$ is given by:

$$
\kappa^{j}=c_{1} \beta^{-1}\left(\sum_{i=0}^{j} L^{-i \varepsilon}\right),
$$

where $c_{1}$ is a sufficiently small constant (so $G^{j}(X, \varphi)$ is integrable with respect to $\left.d \mu_{\beta C}\right)$. Note that $\kappa^{0}=c_{1} \beta^{-1}$ and $\kappa^{0} \leqq \kappa^{j} \leqq 2 \kappa^{0}$.

For the large set regulator we take

$$
\Gamma^{j}(X)=\gamma(X) \Gamma(X) \equiv\left(\gamma^{|X|}\right)\left(A^{|X|} \Theta(X)\right)
$$

with constants $A=2 L^{3}$ and $\gamma=2^{1 / 16}$. The function $\Theta$ is defined by

$$
\Theta(X)=\inf _{\tau} \prod_{b \in \tau} \theta(|b|),
$$

where the infimum is over trees $\tau$ connecting the centers of the blocks of $X$ and where $\theta$ satisfies $\theta(1)=1$ and $\theta(\{s / L\}) \leqq\left(2 L^{3}\right)^{-1} \theta(s)(\{x\}$ is the smallest integer greater than or equal to $x$ ). For definiteness we define

$$
\theta(s)=\left(2 L^{3}\right)^{n+1}, \quad L^{n}<s \leqq L^{n+1}, \quad n \geqq 0 .
$$


Finally we define

$$
\mathbf{h}^{j}=h^{j}(1,1,1),
$$

where

$$
h^{j}=c_{2} \beta\left(1-\sum_{i=1}^{j} L^{-i \varepsilon / 2}\right)
$$

and $c_{2}$ is a constant to be specified (see Sect. 6). We have $h^{0}=c_{2} \beta$ and $h^{0} / 2<h^{j} \leqq h^{0}$.

The main result is:

Theorem 5.1. For any $\beta>8 \pi$, let $L$ be sufficiently large (depending on $\beta$ ), and suppose $|z|$ and hence $\delta^{0} \equiv 6 A|z| e^{h^{0}}$ are sufficiently small (depending on $L$ and $\beta$ ). Then for $0 \leqq j \leqq N$ we have $K^{j} \in \mathscr{K}_{G^{j}, \Gamma^{j}, h^{j}}$ and for $\delta^{j} \equiv L^{-j \varepsilon} \delta^{0}$,

$$
\begin{gathered}
\left\|K^{j}\right\|_{G^{j}, \Gamma^{j}, \mathbf{h}^{j}} \leqq \delta^{j} \\
\left|\delta E^{j}\right| \leqq \delta^{j} \\
\left|\delta \sigma^{j}\right| \leqq \beta\left(h^{j}\right)^{-2} \delta^{j}
\end{gathered}
$$

Remark. As a consequence $\left\|K^{j}\right\|_{G^{\infty}, \Gamma^{\infty}, \mathbf{h}^{\infty}} \leqq \delta^{j}$. This gives the precise sense in which $K^{j} \rightarrow 0$ as $j \rightarrow \infty$.

Proof. At any stage the bound on $K^{j}$ implies the bounds on $\delta E^{j}$ and $\delta \sigma^{j}$. Indeed by bounds like (3.11), (3.12) we have:

$$
\begin{gathered}
\left|\delta E^{j}\right| \leqq\left\|k^{j}(q=0)\right\|_{G^{j}, \Gamma^{j}, \hat{\mathrm{h}}^{j},} \\
\left|\delta \sigma^{j}\right| \leqq \beta\left(h^{j}\right)^{-2}\left\|k^{j}(q=0)\right\|_{G^{j}, \Gamma^{j}, \hat{\mathrm{h}}^{j}}
\end{gathered}
$$

and by (4.4) and the bound on $K^{j}$

$$
\left\|k^{j}(q)\right\|_{G^{j}, \Gamma^{j}, \hat{\mathbf{h}}^{j}} \leqq \exp \left(B h^{j}|q|\right) \delta^{j},
$$

which gives the result (we have suppressed $1_{X \in \mathcal{G}}$ from the notation).

The bound for $K^{0}$ follows by Lemma 3.4. We now assume the bound on $K^{j}$ and use it to prove the bound on $K^{j+1}$ following the sequence $K^{j} \rightarrow K^{\#} \rightarrow K^{*} \rightarrow K^{j+1}$.

To estimate $K^{\#}$ (the result of the fluctuation integral) we introduce

$$
\begin{aligned}
G^{\#}(X, \varphi) & =\exp \left(1 / 2 \kappa^{j} \sum_{0<|\alpha| \leqq s} L^{2|\alpha|-2}\left\|\partial^{\alpha} \varphi\right\|_{X_{1 / 3}}^{2}\right), \\
\Gamma^{\#}(X) & =\Gamma(X), \\
\mathbf{h}^{\#} & =h^{j+1}(1,1 / 2,1 / 4) .
\end{aligned}
$$

\section{Proposition 1.}

(i)

(ii)

$$
\left\|K^{\#}\right\|_{G^{\#}, \Gamma^{\#}, \mathrm{~h}^{\#}} \leqq \delta^{j}
$$



We postpone the proof of Chap. 6 where $\beta^{*}$ is defined; roughly it is $\beta \log L / 2 \pi$. 
The factor $\exp \left(-(|q|-1 / 2) \beta^{*}\right)$ gives the contraction for the $q \neq 0$ Fourier modes for small sets.

To estimate $K^{*}$ (the result of extraction and reblocking) we introduce

$$
\begin{aligned}
G^{*}(U \varphi) & =\exp \left(1 / 2 \kappa^{j+1} \sum_{0<|\alpha| \leqq s} L^{2|\alpha|-2}\left\|\partial^{\alpha} \varphi\right\|_{U_{3}}^{2}\right), \\
\Gamma^{*}(U) & =\gamma(U) \Gamma(U), \\
\mathbf{h}^{*} & =h^{j+1}\left(1, L^{-1}, L^{-2}\right) .
\end{aligned}
$$

Here $U$ is a union of $L$-blocks and in $\Gamma^{*}(U)$ the $|U|$ refers to the number of L-blocks, etc.

\section{Proposition 2.}

$$
\left\|K^{*}\right\|_{G^{*}, \Gamma^{*}, \mathrm{~h}^{*}} \leqq \delta^{j+1} .
$$

The proof is postponed to Chap. 7. The issue is to find contractive factors for large sets and for $q=0$ modes for small sets.

We complete the proof of Theorem 5.1 by scaling the above bound to obtain:

$$
\left\|K^{j+1}\right\|_{G^{j+1}, \Gamma^{j+1}, h^{j+1}} \leqq \delta^{j+1} \text {. }
$$

(Actually we get $G^{*}(L X, R \varphi)$ which is $G^{j+1}(X, \varphi)$ with $X_{3 / L}$ instead of $X_{1 / 6}$. Since for $L$ large $X_{3 / L} \subset X_{1 / 6}$ the result follows.)

\section{Proof of Proposition 1: Fluctuation Integral}

We consider the one parameter family $K(t), 0 \leqq t \leqq 1$ defined by the fluctuation integral

$$
\mathscr{E} \mathrm{xp}(\square+K(t))=\mu_{t \beta C} *\left[\mathscr{E} \mathrm{xp}\left(\square+K^{j}\right)\right]
$$

By assuming inductively that Theorem 5.1 holds at the $j^{\text {th }}$ level, we have estimates on $K(0) \equiv K^{j}$, and we seek to produce bounds on $K^{\#} \equiv K(1)$. The proof follows [BY, Theorem B]. Now $K(t)$ is the solution of an integral equation:

$$
K(t)=\mu_{t \beta C} * K(0)+1 / 2 \int_{0}^{t} \mu_{(t-s) \beta C} *\left(K_{1}(s), \beta C K_{1}(s)\right) d s
$$

We use the notation

$$
\left(K_{1}, C K_{1}\right)=\int d \xi d \xi^{\prime} C\left(\xi, \xi^{\prime}\right) K_{1}(\xi) \circ K_{1}\left(\xi^{\prime}\right)
$$

with $\xi=(x, \omega) \in \Lambda \times \Omega, \Omega=\Omega_{0} \times \Omega_{1} \times \Omega_{2}, \int d \xi=\int d x \sum_{\omega}$, and $K_{1}(\xi)=\delta K / \delta \psi(\xi)$.

Part (i): Part (i) of Proposition 1 is proved exactly as in Brydges and Yau by considering the function (analytic in $h$ )

$$
k(t, h)=\|K(t)\|_{g(t), \Gamma^{j}, \mathrm{~h}},
$$

where $\mathbf{h}=(h, h, h)$ and

$$
g(t, X)=\left[\gamma^{|X|} G^{\#}(X)\right]^{t}\left[G^{j}(X)\right]^{1-t}, 0 \leqq t \leqq 1 .
$$


Note that $g(1)=\gamma G^{\#}, \Gamma^{j}=\gamma^{-1} \Gamma^{\#}$ and $\mathbf{h}^{\#} \leqq \mathbf{h}^{j+1}$ so

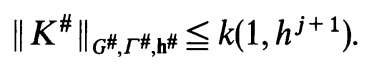

Therefore, the bound of part (i) follows if we prove

$$
k\left(1, h^{j+1}\right) \leqq k\left(0, h^{j}\right) \equiv\left\|K^{j}\right\|_{G^{j}, \Gamma^{j}, \mathbf{h}^{j}}
$$

It is essential that the interpolated large field regulators $g(t)$ satisfy the homotopy property:

$$
\mu_{(t-u) \beta C} * g(u) \leqq g(t) \text { for all } 0 \leqq u \leqq t \leqq 1 .
$$

The proof of this result given in [BY, Proposition 9.1] applies here provided $\kappa^{j}$ is sufficiently small; in particular we need

$$
\kappa^{j}<\left(2 \beta\|C\|_{-s \rightarrow s}\right)^{-1} \text {. }
$$

Lemma A.2 in the appendix shows that $\|C\|_{-s \rightarrow s}$, the norm of the fluctuation covariance as a linear operator $C: \mathscr{H}^{\prime}{ }_{s}(\Lambda) \rightarrow \mathscr{H}_{s}^{\prime}(\Lambda)$, is bounded by $\mathcal{O}(1)$ (the prime indicates no zeroth order derivatives). Thus we can satisfy (6.5) by taking $c_{1}$ small enough (recall $\kappa^{j} \leqq \kappa^{0}=2 c_{1} \beta^{-1}$ ).

The homotopy property leads to the following result [cf. BY, Proposition 8.3)]: the power series for $k(t, h)$ is term by term dominated by the solution of

$$
k^{*}(t, h)=k(0, h)+\beta\|C\| \int_{0}^{t} d s\left(\frac{\partial k^{*}(s, h)}{\partial h}\right)^{2}
$$

or equivalently,

$$
\frac{\partial k^{*}}{\partial t}=\beta\|C\|\left(\frac{\partial k^{*}}{\partial h}\right)^{2}, \quad k^{*}(0, h)=k(0, h) .
$$

In our setup we have an extra derivative $\left(\partial / \partial \psi_{0}\right)$ not present in [BY]. This does not affect the proof, but it does modify $\|C\|$ :

$$
\|C\|=\sup _{\Delta \in \Lambda} \sum_{\Delta^{\prime} \in \Lambda} \sup _{\substack{x \in \Delta, x^{\prime} \in \Delta^{\prime} \\ \alpha, \alpha^{\prime} \in \Omega}}\left|\partial^{\alpha} \partial^{\alpha^{\prime}} C\left(x-x^{\prime}\right)\right| \theta\left(d\left(\Delta, \Delta^{\prime}\right)\right) .
$$

Lemma 8.4 of [BY] applies to (6.7) and shows that the solution $k^{*}$ is analytic in $0 \leqq t \leqq 1$ and $h$ near the origin, and that

$$
k^{*}\left(1, h_{1}\right) \leqq k^{*}\left(0, h_{0}\right)
$$

provided $h_{0}, h_{1}$, and $k^{*}\left(0, h_{0}\right)$ are chosen such that $0<h_{1}<h_{0}$ and

$$
k^{*}\left(0, h_{0}\right) \leqq \frac{\left(h_{0}-h_{1}\right)^{2}}{16 \beta\|C\|} .
$$

With $h_{0}=h_{j}$ and $h_{1}=h_{j+1}$ the bound (6.9) gives the required bound (6.3). The condition (6.10) holds if

$$
\delta^{j} \leqq \frac{\left(h^{j}-h^{j+1}\right)^{2}}{16 \beta\|C\|} .
$$

This is true if $\delta^{0}$ is small: Lemma A.2 says $\|C\| \leqq \mathcal{O}(1) L^{9}$ and moreover, from (5.7) 
we have $h^{j}+h^{j+1}=\mathcal{O}(1) \beta L^{-j \varepsilon / 2}$. Thus the bound holds provided we choose $\delta^{0} \leqq \mathcal{O}(1) \beta L^{-9}$.

Part (ii): The analysis for the small set part $K(t) 1_{X \in \mathscr{S}}$ is simplified by noting that the circle product of two activities vanishes, $\left(K \circ K^{\prime}\right)(X)=0$, whenever $X$ is a connected set. This means the non-linear term in (6.2) drops out if $X$ is a small set:

$$
K^{\#}(X)=\mu_{\beta C} *\left(K^{j}(X)\right), \quad X \in \mathscr{S} .
$$

We are concerned with the Fourier components of $K^{\#}$. By the definition of $k^{\#}$,

$$
\begin{aligned}
k^{\#}(X, q, \hat{\psi}) & =(2 \pi)^{-1} \int_{-\pi}^{\pi} d \Phi e^{-i q \Phi} K^{\#}\left(X, \Phi+H \psi_{1}, \hat{\psi}\right) \\
& =(2 \pi)^{-1} \int d \mu_{\beta C}(\zeta) \int_{-\pi}^{\pi} d \Phi e^{-i q \Phi} K^{j}\left(X, \Phi+H \psi_{1}+\zeta, \hat{\psi}+\widehat{\zeta}\right) \\
& =(2 \pi)^{-1} \int d \mu_{\beta C}(\zeta) \int_{-\pi}^{\pi} d \Phi e^{-i q \Phi} K^{j}\left(X, \Phi+\zeta(x)+H\left(\psi_{1}+\partial \zeta\right), \hat{\psi}+\hat{\zeta}\right),
\end{aligned}
$$

where $\hat{\zeta}=(\partial \zeta, \partial \partial \zeta)$ and we have used

$$
\zeta=\zeta(x)+H(\partial \zeta)
$$

Because $K^{j}$ is periodic in $\Phi$, we can shift the integration variable $\Phi \rightarrow \Phi-\zeta(x)$ to produce the desired fluctuation integral:

$$
\begin{aligned}
k^{\#}(X, q, \hat{\psi}) & =(2 \pi)^{-1} \int d \mu_{\beta C}(\zeta) \int_{-\pi}^{\pi} d \Phi e^{-i q(\Phi-\zeta(x))} K^{j}\left(X, \Phi+H\left(\psi_{1}+\partial \zeta\right), \hat{\psi}+\widehat{\zeta}\right) \\
& =\int d \mu_{\beta C}(\zeta) e^{i q \zeta(x)} k^{j}(X, q, \hat{\psi}+\widehat{\zeta}) .
\end{aligned}
$$

The case $q=0$ is easily dealt with:

$$
\begin{aligned}
\left\|k^{\#}(q=0)\right\|_{G^{\#}, \Gamma^{\#}, \hat{\mathbf{h}}^{\#}} & \leqq\left\|\mu_{\beta C} * k^{j}(q=0)\right\|_{g(1), \Gamma^{j}, \hat{\mathbf{h}}^{j}} \\
& \leqq\left\|k^{j}(q=0)\right\|_{g(0), \Gamma^{j}, \hat{\mathbf{h}}^{j}} \\
& =\left\|k^{j}(q=0)\right\|_{G^{j}, \Gamma^{j}, \hat{\mathbf{h}}^{j}} \\
& \leqq \delta^{j} .
\end{aligned}
$$

The first inequality requires $\hat{\mathbf{h}}^{\#} \leqq \hat{\mathbf{h}}^{j}$. The second inequality follows from

$$
\left\|\mu_{(t-u) \beta C} * A\right\|_{g(t)} \leqq\|A\|_{g(u)}, \quad 0 \leqq u \leqq t \leqq 1,
$$

which holds for any functional $A(X, \psi)$ or derivative as a consequence of the homotopy property (6.4). The last inequality is (5.13).

When $q \neq 0$, we use analyticity to make a complex shift $\zeta \mapsto \zeta \pm$ if in the fluctuation integral for $k^{\#}(q)$ :

$$
\begin{aligned}
k^{\#}(X, q, \hat{\psi})= & e^{-|q| f(x)+1 / 2\left(f,(\beta C)^{-1} f\right)} \\
& \cdot \int d \mu_{\beta C}(\zeta) e^{ \pm i\left(f,(\beta C)^{-1} \zeta\right)} e^{i q \zeta(x)} k^{j}(X, q, \hat{\psi}+\hat{\zeta} \pm i \hat{\mathbf{f}}) .
\end{aligned}
$$

The sign of the shift depends on the sign of $q$. The function $f$ is chosen to minimize $\left.-f(x)+1 / 2(f, \beta C)^{-1} f\right)$ :

$$
f(y)=\beta C(y, x)
$$


and then

$$
\begin{gathered}
f(x)=\beta^{*} \equiv \beta C(0,0) \\
1 / 2\left(f,(\beta C)^{-1} f\right)=1 / 2 \beta^{*} .
\end{gathered}
$$

This choice of $f$ produces optimal bounds on $k^{\#}(q)$ for $|q|=1$.

Taking derivatives and estimating the integral we find

$$
\left\|k_{\mathbf{n}}^{\#}\left(X, q, \psi_{\varphi}\right) 1_{\Delta}\right\| \leqq e^{-(|q|-1 / 2) \beta^{*}} \mu_{\beta C} *\left\|k_{\mathbf{n}}^{j}\left(X, q, \cdot+\hat{\psi}_{\varphi} \pm i \hat{\mathbf{f}}\right) 1_{\Delta}\right\| .
$$

Using the homotopy property (6.4) again yields

$$
\begin{aligned}
& \left\|k_{\mathbf{n}}^{\#}(X, q)\right\|_{g(1)} \leqq e^{-(|q|-1 / 2) \beta^{*}}\left\|k_{\mathbf{n}}^{j}(X, q, \cdot \pm i \hat{\mathbf{f}})\right\|_{g(0)},
\end{aligned}
$$

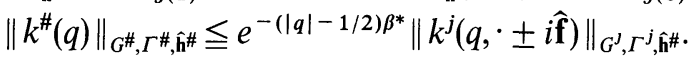

But Lemma 3.2 implies

$$
\left\|k^{j}(q, \cdot \pm i \hat{\mathbf{f}})\right\|_{G^{j}, \Gamma^{j}, \hat{\mathrm{h}}^{\sharp}} \leqq\left\|k^{j}(q)\right\|_{G^{j}, \Gamma^{j}, \hat{\mathrm{h}}^{\sharp}+\hat{\mathrm{N}}_{\mathbf{f}}}
$$

where $\hat{\mathbf{N}}_{\mathbf{f}}=\left(|\partial f|_{\infty},|\partial \partial f|_{\infty}\right)$. By Lemma A.2, $|\partial f|_{\infty},|\partial \partial f|_{\infty}<\mathcal{O}(1) \cdot \beta$, and so for $c_{2}$ large enough

$$
\hat{\mathbf{h}}^{\#}+\hat{\mathbf{N}}_{\mathbf{f}} \leqq \hat{\mathbf{h}}^{j}
$$

The result now follows by (5.13).

\section{Proof of Proposition 2: Extraction and Reblocking}

It remains to pin down the definition of $K^{*}$, and to bound it using the bounds on $K^{\#}$. The proof is an extension of [BY, Theorem A], with extra care needed to treat the various Fourier modes.

The overall requirement on the reblocked activities is that

$$
K^{*}(U) \doteq \sum_{\left\{X_{i}\right\} \rightarrow U} h\left(\cup_{i} X_{i}\right) \prod_{i} J\left(X_{i}\right)
$$

that is, the equation holds for fields $\psi=\psi_{\varphi} \in \mathscr{M}_{0}$. The sum is over collections of disjoint sets $X_{i}$ such that (i) the Mayer graph on $\left\{\bar{X}_{i}\right\}$ (i.e. the graph on $\left\{\bar{X}_{i}\right\}$ of lines joining overlapping pairs $\bar{X}_{i}, \bar{X}_{j}$ ) is connected, (ii) $\cup_{i} \bar{X}_{i}=U$, where $\bar{X}_{i}$ denotes the smallest union of $L$-blocks which contains $X_{i}$, a union of unit blocks. The quantities $h, R, J$ are defined in terms of $F$ (which is given by Eq. 2.18) by:

$$
\begin{gathered}
h(X, \hat{\psi})=\prod_{Y: Y \cap X \neq \varnothing} \exp -F(Y, \widehat{\psi}), \\
R(X, \hat{\psi})=\exp F(X, \widehat{\psi})-1, \\
J(X, \psi)=K^{\#}(X, \psi)-\sum_{\left\{X_{i}\right\} \rightarrow X} \prod_{i} R\left(X_{i}\right),
\end{gathered}
$$

where in (7.4) the sum is over collections of distinct sets $X_{i}$ such that (i) the Mayer graph is connected, (ii) $\cup_{i} X_{i}=X$.

$K^{*}$ is defined as a sum $K_{1}^{*}+K_{2}^{*}$. The first term $K_{1}^{*}$ is chosen to include all contributions which are first order in the Fourier modes of $K^{\#}$. It will be small due to renormalization subtractions for $k^{\sharp}(q=0)$ and the overall smallness 
of $k^{\#}(q \neq 0)$. The remainder $K_{2}^{*}$ contains only terms which are either higher order in $K^{\#}$ or have factors of $K^{\#}$ on large sets.

For unions $U$ of $L$-blocks, we define

$$
\begin{gathered}
K_{1}^{*}(U)=\sum_{X \in \mathscr{S}: \bar{X}=U} h(X) I(X), \\
K_{2}^{*}(U)=\sum_{X: \bar{X}=U} h(X)\left[\left(K^{\#}(X)-R(X)\right) 1_{X \notin \mathscr{S}}-R^{+}(X)+J^{+}(X)\right] .
\end{gathered}
$$

The quantity $I$ is defined in the next paragraph in such a way that $I \doteq K^{\#}-R$,

$$
\begin{aligned}
& R^{+}(X)=\sum_{\substack{\left\{X_{i} i \rightarrow X \\
\geqq 2\right. \text { sets }}} \prod_{i} R\left(X_{i}\right), \\
& J^{+}(X)=\sum_{\substack{\left\{X_{i}\right\} \rightarrow X \\
\geqq 2 \operatorname{sets}}} \prod_{i} J\left(X_{i}\right),
\end{aligned}
$$

where (7.7) is summed as in (7.4) and (7.8) is summed as in (7.1) except condition (ii) becomes $\cup X_{i}=X$. With $K_{1}^{*}$ and $K_{2}^{*}$ chosen in this way, the reader may check that (7.1) is indeed satisfied.

We define for $X \in \mathscr{S}$

$$
\begin{gathered}
I(X, \psi)=\sum_{q \in \mathbf{Z}} e^{i q \psi_{0}(x)} i(X, q, \hat{\psi}) \\
i(X, q=0, \hat{\psi})=\left[k^{\#}(X, q=0, \hat{\psi})-\delta E^{j}(X)-\frac{1}{2} \sum_{\mu, v} \int Q_{\mu v}(X ; x, y) \psi_{\mu}(x) \psi_{v}(y) d x d y\right] \\
+\frac{1}{2} \sum_{\mu, v}|X|^{-1} \int_{X} d z\left[\int Q_{\mu v}(X ; x, y) W_{\mu \nu}(\hat{\psi} ; x, y, z) d x d y\right] \\
+[F(X, \hat{\psi})-R(X, \hat{\psi})] \\
\quad i(X, q \neq 0, \hat{\psi})=k^{\#}(X, q \neq 0, \hat{\psi}) .
\end{gathered}
$$

Here

$$
Q_{\mu \nu}(X ; x, y)=k_{2,0}^{\#}(X, q=0, \hat{\psi}=0 ; x, \mu, y, v)
$$

and

$$
\begin{aligned}
W_{\mu v}(\hat{\psi} ; x, y, z)= & \int_{0}^{1} d s \sum_{\sigma}\left[(x-z)_{\sigma} \psi_{\mu \sigma}(z+s(x-z)) \psi_{v}(z+s(y-z))\right. \\
& \left.+(y-z)_{\sigma} \psi_{\mu}(z+s(x-z)) \psi_{v \sigma}(z+s(y-z))\right] .
\end{aligned}
$$

Since

$$
W_{\mu v}(\hat{\psi} ; x, y, z) \doteq \psi_{\mu}(x) \psi_{v}(y)-\psi_{\mu}(z) \psi_{v}(z)
$$

and

$$
\delta \sigma_{\mu \nu}^{j}(X)=-\beta|X|^{-1} \int Q_{\mu v}(X ; x, y) d x d y \quad \text { and } \quad K^{\#^{\prime \prime}} \doteq K^{\#}
$$

we have that $I \doteq K^{\#}-R$. In addition, this definition has the desired feature that it can be bounded directly in terms of the Fourier modes $k^{\#}(q)$, rather than $K^{\#}$. 


\section{Lemma 7.1.}

(i)

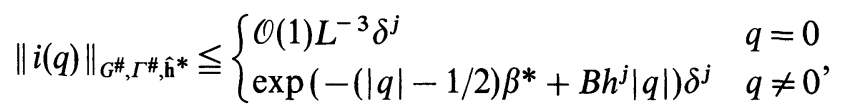

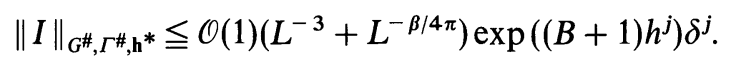

Proof. (i) The bound for $q \neq 0$ follows directly from the bound on $k^{\#}(q)$ since $\mathbf{h}^{*}<\mathbf{h}^{\#}$.

The bound for $q=0$ uses the fact that $i(q=0)$ is an irrelevant functional, i.e. that the derivatives $i_{\mathbf{n}}(X, q=0, \psi=0)$ vanish if $\operatorname{dim} \mathbf{n} \equiv n_{1}+2 n_{2} \leqq 2$. This of course is the point of performing the extractions.

Lemma 4.3 of $[\mathrm{BY}]$, which deals with functionals whose low order derivatives vanish, is applicable here since $\left(\kappa_{j} h_{j+1}^{2}\right)^{-1} \leqq \mathcal{O}(1)$ and implies

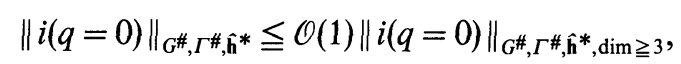

where "dim $\geqq 3$ " indicates which low order derivatives are omitted from the norm. By changing $\mathbf{h}^{*}$ to $\mathbf{h}^{\#}$ in the $\operatorname{dim} \geqq 3$ norm, we can extract a factor of $(2 / L)^{3}$,

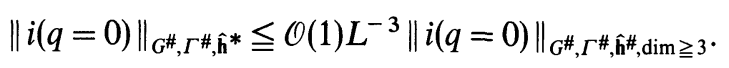

Finally, we need to bound the norm on the right by $\mathcal{O}(1)\left\|k^{\#}(q=0)\right\|_{G^{\#}, \Gamma^{\#}, \hat{h}^{\#} \text {. This }}$ requires estimating each of the three terms of (7.10) exactly as done in [BY, Lemmas 4.2, 4.4].

(ii) Following the proof of Lemma 4.2 and using the bounds of (i) for $i(q)$, we have

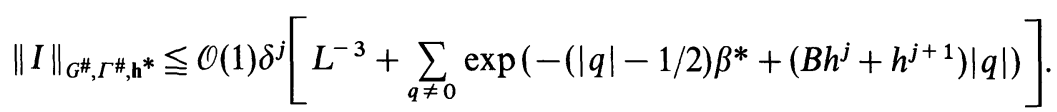

Lemma A.3 in the appendix shows that for $L$ sufficiently large,

$$
\left|\beta^{*}-\beta \log L / 2 \pi\right|<1 \text {, }
$$

and hence $\beta^{*}>(B+1) h^{j}>B h^{j}+h^{j+1}$. Then the series converges and is dominated by $\mathcal{O}(1) \exp \left(-\beta^{*} / 2+(B+1) h^{j}\right)$ (the $|q|=1$ term). By (7.12) this is bounded by $\mathcal{O}(1) L^{-\beta / 4 \pi} \exp \left((B+1) h^{j}\right)$ to complete the proof.

\section{Lemma 7.2.}

(i)

$$
\left\|K_{1}^{*}\right\|_{G^{*}, \Gamma^{*}, \mathbf{h}^{*}} \leqq \mathcal{O}(1)\left(L^{-1}+L^{2-\beta / 4 \pi}\right) \exp \left((B+1) h^{j}\right) \delta^{j}
$$

$$
\left\|K_{2}^{*}\right\|_{G^{*}, \Gamma^{*}, \mathrm{~h}^{*}} \leqq \mathcal{O}(1) L^{-1} \delta^{j} .
$$

Remarks. (a) This finishes the proof of Proposition 2. We have for $L$ sufficiently large:

$$
\left\|K^{*}\right\|_{G^{*}, \Gamma^{*}, \mathbf{h}^{*}} \leqq \mathcal{O}(1) L^{-2 \varepsilon} \exp \left((B+1) h^{j}\right) \delta^{j} \leqq L^{-\varepsilon} \delta^{j}=\delta^{j+1}
$$

(b) In the proof we need the definitions

$$
\begin{gathered}
\|K\|_{G, \Gamma, \mathbf{h}}^{(1)}=\sum_{\mathbf{n}} \mathbf{h}^{\mathbf{n}} / \mathbf{n} ! \sum_{X: \bar{X}_{\ni \Delta}} \Gamma(\bar{X})\left\|K_{\mathbf{n}}(X)\right\|_{G}, \\
|K|_{G, \Gamma, \mathbf{h}}^{(1)}=\sum_{\mathbf{n}} \mathbf{h}^{\mathbf{n}} / \mathbf{n} ! \sup _{X} \Gamma(\bar{X})\left\|K_{\mathbf{n}}(X)\right\|_{G} .
\end{gathered}
$$


Proof. (i) We have (cf. [BY, Eq. (7.4)]),

$$
\left\|K_{1}^{*}\right\|_{G^{*}, \Gamma^{*}, \mathbf{h}^{*}} \leqq|h|_{\delta G, \gamma^{-1}, \mathbf{h}^{*}}^{(1)}\|I\|_{G^{\#}, \gamma^{2} \Gamma^{\#, \mathbf{h}^{*}}}^{(1)} .
$$

where $\Gamma^{*} \equiv \gamma \Gamma^{\#}$ and $\delta G \equiv G^{*}\left(G^{\#}\right)^{-1}$.

But $|h|^{(1)} \leqq 4\left[\mathrm{BY}\right.$, Lemma 6.1] provided $\delta^{0}$ is sufficiently small $\left(\delta^{0} \leqq O\left(L^{-2} \kappa_{0} h_{0}^{2}\right)\right)$. Furthermore since $\gamma^{2} \leqq 2^{1 / 4}$ we have by [BY, Lemma 3.1]:

$$
\|I\|_{G^{\#}, \gamma^{2} \Gamma^{\#}, \mathbf{h}^{*}}^{(1)} \leqq 18 L^{2}\|I\|_{G^{\#}, \Gamma^{\#, h^{*}},}
$$

and the result follows by Lemma 7.1 (ii).

(ii) The terms in $K_{2}^{*}$ are estimated similarly by

$$
\left\|K_{2}^{*}\right\|_{G^{*}, \Gamma^{*}, \mathrm{~h}^{*}} \leqq\left\|K_{2}^{*}\right\|_{G^{*}, \Gamma^{*}, \mathrm{~h}^{\#}} \leqq 4\left\|\left(K^{\#}-R\right) 1_{X \notin \mathscr{S}}-R^{+}+J^{+}\right\|_{G^{\#, \gamma^{2}} \Gamma^{\#, \mathrm{~h}^{*}}}^{(1)}
$$

For the first term, the large set restriction gives a contractive factor,

$$
\begin{aligned}
& \left\|\left(K^{\#}-R\right) 1_{X \notin \mathscr{S}}\right\|_{G^{\#}, \gamma^{2} \Gamma^{\#, h^{\#}}}^{(1)} \leqq 18 L^{-1}\left\|K^{\#}-R\right\|_{G^{\#}, \Gamma^{\#}, \mathrm{~h}^{\#}} \\
& \leqq \mathcal{O}(1) L^{-1} \delta^{j} \text {. }
\end{aligned}
$$

Here we have used [BY, Lemma 3.1] for the first step and the bound $\|R\| \leqq \mathcal{O}(1) L^{-1} \delta_{j}$ from [BY, Lemma 4.2] combined with the bound on $\left\|K^{\#}\right\|$ for the second step.

The second and third terms in (7.16) contract because they are higher order. By [BY, Lemmas 3.1 and 5.2], if $\delta^{0}$ is sufficiently small $\left(\leqq \mathcal{O}\left(L^{-3}\right)\right)$,

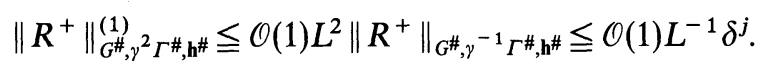

Similarly, for the third term of (7.16), [BY, Lemma 7.1] implies

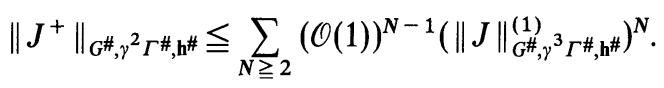

Moreover, since $J=K^{\#}-R-R^{+}$,

$$
\|J\|_{G^{\#}, \gamma^{3} \Gamma^{\#, h^{\#}}}^{(1)} \leqq \mathcal{O}(1) L^{2}\|J\|_{G^{\#,}, \gamma^{-1} \Gamma^{\#, h^{\#}}} \leqq \mathcal{O}(1) L^{2} \delta^{j},
$$

where in the first step we decrease $\Gamma$ by $\gamma^{4}=2^{1 / 4}$, the largest factor allowed by [BY, Lemma 3.1]. Therefore, provided $\delta^{0} \leqq \mathcal{O}\left(L^{-5}\right)$,

$$
\left\|J^{+}\right\|_{G^{\#}, \gamma^{2} \Gamma^{\#, h^{\#}}}^{(1)} \leqq \mathcal{O}(1) L^{-1} \delta^{j} .
$$

Concluding Remark. We explain the sense in which we have established Fig. 1. The conditions on the parameters $(\beta, z)$ in Theorem 5.1 are satisfied if $z \leqq \mathcal{O}(1) L^{-n} \exp \left(-1 / 2 c_{2} \beta\right)$ for suitable $n$. Given this, $z$ must be taken exponentially small as $\beta \rightarrow \infty$. As $\beta \rightarrow 8 \pi$ we must have $L \rightarrow \infty$ to ensure that the bound (7.13) holds, and thus $z \rightarrow 0$ in this limit as well. Thus the constraints on $(\beta, z)$ describe a region roughly like that of Fig. 1.

The flow of the renormalization group transformations can be tracked by two effective parameters. Of course the actual flow is in an infinite dimensional space. For example we might define

$$
\begin{aligned}
\beta_{j}^{-1} & =\left.\frac{d}{d\left(p^{2}\right)}\left(\beta \tilde{v}^{j}(p)\right)^{-1}\right|_{p^{2}=0} \\
& =\beta^{-1}\left(1+\sigma^{j}\right), \\
z_{j} & =\left\|K^{j}\right\|_{G^{\infty}, \Gamma, \mathbf{h}^{\infty}},
\end{aligned}
$$


and then $\beta_{j}=\beta\left(1+\sigma^{j}\right)^{-1}$ converges to $\beta_{\infty}=\beta\left(1+\sigma^{\infty}\right)^{-1}$ and $z_{j} \rightarrow 0$ as $j \rightarrow \infty$. The points $\left(\beta_{j}, z_{j}\right)$ would lie on the indicated flow lines in Fig. 1.

\section{A. Appendix: Lemmas on $C$}

Here we collect properties of the fluctuation covariance

$$
C(x)=|\Lambda|^{-1} \sum_{p \in \Lambda^{*}} e^{i p \cdot x} p^{-2} \int_{L^{2}}^{1} d s \frac{\partial}{\partial s}\left(e^{s^{2} p^{4}}+\sigma\right)^{-1},
$$

which are used throughout the paper. In what follows we take the torus $\Lambda$ of side $L^{N}, N>0$ and assume $|\sigma| \leqq 1 / 2$.

Lemma A.1. For each multiindex $\alpha,|\alpha| \leqq s$ and integer $n=0,1, \ldots, M$, there is $a$ constant $A_{s, M}$ such that

$$
\left|\partial^{\alpha} C(x)\right| \leqq\left\{\begin{array}{lll}
A_{s, M} L^{2 n-|\alpha|}\left(1+|x|^{2 n}\right)^{-1} & \text { if } & |\alpha|<2 n \\
A_{s, M} \log L\left(1+|x|^{2 n}\right)^{-1} & \text { if } & |\alpha|=2 n \\
A_{s, M}\left(1+|x|^{2 n}\right)^{-1} & \text { if } & |\alpha|>2 n
\end{array}\right.
$$

Proof. We write

$$
|x|^{2 n} \partial^{\alpha} C(x)=-2 \int_{L^{2}}^{1} d s|\Lambda|^{-1} \sum_{p \in \Lambda^{*}} e^{i p \cdot x}|x|^{2 n}(i p)^{\alpha} p^{-2} s p^{4}\left(e^{s^{2} p^{4}}+\sigma\right)^{-2} .
$$

When we rescale $p \rightarrow p^{\prime}=s^{1 / 2} p$ we find

$$
\begin{aligned}
\sup _{x \in \Lambda}|x|^{2 n}\left|\partial^{\alpha} C(x)\right| \leqq & 2 \int_{L^{2}}^{1} d s s^{n-|\alpha| / 2-1} \\
& \cdot \sup _{x \in\left(s^{-1 / 2} \Lambda\right)}\left|s^{-1 / 2} \Lambda\right|^{-1}\left|\sum_{p^{\prime} \in\left(s^{-1 / 2} \Lambda\right)^{*}} e^{i p^{\prime} \cdot x}\left(x^{2}\right)^{n}\left(i p^{\prime}\right)^{\alpha}\left(p^{\prime}\right)^{2}\left(e^{p^{\prime 4}}+\sigma\right)^{-2}\right| .
\end{aligned}
$$

The supremum over $x \in\left(s^{-1 / 2} \Lambda\right)$ is $\mathcal{O}(1)$ for any value $1 \leqq s \leqq L^{2}$ and any volume $\left|s^{-1 / 2} \Lambda\right| \geqq 1$. To see this, note that

$$
\left.|x|^{2} \leqq \mathcal{O}(1) \varepsilon^{-2} \sum_{\mu=1,2}\left(1-\cos \varepsilon x_{\mu}\right)\right), \quad \varepsilon=2 \pi s^{1 / 2} L^{-N}
$$

for any $x \in s^{-1 / 2} \Lambda$. Insertion of this inequality and summation by parts on the momentum lattice leads to

$$
\begin{aligned}
& \sup _{x \in\left(s^{-1 / 2} \Lambda\right)}\left|s^{-1 / 2} \Lambda\right|^{-1}\left|\sum_{p \in\left(s^{-1 / 2} \Lambda\right)^{*}} e^{i p \cdot x}\left(x^{2}\right)^{n}(i p)^{\alpha}\left(p^{2}\right)\left(e^{p^{4}}+\sigma\right)^{-2}\right| \\
& \leqq \mathcal{O}(1)\left|s^{-1 / 2} \Lambda\right|^{-1}\left|\sum_{p \in\left(s^{-1 / 2} \Lambda\right)^{*}}\left(-\Delta_{p}\right)^{n}(i p)^{\alpha}\left(p^{2}\right)\left(e^{p^{4}}+\sigma\right)^{-2}\right| \\
& \leqq \mathcal{O}(1)\left[\int_{\mathbf{R}^{2}} d p\left|\left(-\partial_{p}^{2}\right)^{n}(i p)^{\alpha}\left(p^{2}\right)\left(e^{p^{4}}+\sigma\right)^{-2}\right|+\mathcal{O}(1)\right] \\
& \leqq \mathcal{O}(1),
\end{aligned}
$$

where $-\Delta_{p}$ is the lattice laplacian. The three bounds of Lemma A.1 now follow by doing the $s$-integral. 


\section{Corollary A.2.}

(i)

(ii)

(iii)

(iv)

$$
|\partial C|_{\infty}<\mathcal{O}(1),
$$

$$
|\partial \partial C|_{\infty}<\mathcal{O}(1)
$$

$$
\|C\|<\mathcal{O}(1) L^{9},
$$

$$
\|C\|_{-s \rightarrow s}<\mathcal{O}(1) .
$$

Proof. Bounds (i) and (ii) are immediate from Lemma A.1 with $n=0$. Starting from the definition (6.8) of $\|C\|$,

$$
\begin{aligned}
\|C\| & =\sup _{\Delta \in \boldsymbol{\Delta}} \sum_{\Delta^{\prime} \in \boldsymbol{\Lambda}} \sup _{\substack{x \in \Delta, x^{\prime} \in \Delta^{\prime} \\
\alpha, \alpha^{\prime} \in \Omega}}\left|\partial^{\alpha} \partial^{\alpha^{\prime}} C\left(x-x^{\prime}\right)\right| \theta\left(d\left(\Delta, \Delta^{\prime}\right)\right) \\
& \leqq \sup _{\Delta \in \boldsymbol{\Lambda}} \sum_{\Delta^{\prime} \in \boldsymbol{\Lambda}}\left[\mathcal{O}(1) L^{2 n}\left(1+d\left(\Delta, \Delta^{\prime}\right)^{2}\right)^{-n}\right]\left[\mathcal{O}(1) L^{3}\left(d\left(\Delta, \Delta^{\prime}\right)^{3}\right]\right.
\end{aligned}
$$

which, for $n=3$ yields bound (iii).

For (iv), $C$ is considered as a linear mapping $C: \mathscr{H}^{\prime}{ }_{-s}(\Lambda) \rightarrow \mathscr{H}_{s}^{\prime}(\Lambda)$ :

$$
\begin{aligned}
\|C\|_{-s \rightarrow s} & \leqq \sup _{p \in \Lambda^{*}} \sum_{\alpha: 1 \leqq|\alpha| \leqq s}\left(p^{\alpha}\right)^{2} \tilde{C}(p) \\
& \leqq \sup _{p \in \Lambda^{*}} \sum_{\alpha: 1 \leqq|\alpha| \leqq s}\left(p^{\alpha}\right)^{2} p^{-2}\left(e^{p^{4}}+\sigma\right)^{-1} \\
& \leqq \mathcal{O}(1),
\end{aligned}
$$

and bound (iv) is proved.

Lemma A.3. For a torus $\Lambda$ of side $L^{N}, N>0$,

$$
C(0)=\frac{\log L}{2 \pi(1+\sigma)}+\mathcal{O}(1) L^{5-N} .
$$

Proof. Write

$$
C(0)=\frac{1}{(2 \pi)^{2}} \int_{\mathbf{R}^{2}} d p \tilde{C}(p)+R
$$

and ignore the error for the moment. Then the first term is

$$
\begin{aligned}
\frac{1}{(2 \pi)^{2}} \int_{\mathbf{R}^{2 .}} d p p^{-2} \int_{L^{2}}^{1} d s \frac{\partial}{\partial s}\left(e^{s^{2} p^{4}}+\sigma\right)^{-1} & =\frac{1}{4 \pi} \int_{L^{2}}^{1} \frac{d s}{s} \int_{0}^{\infty} d \rho \frac{\partial}{\partial \rho}\left(e^{(s \rho)^{2}}+\sigma\right)^{-1} \\
& =\left.\frac{1}{4 \pi} \int_{L^{2}}^{1} \frac{d s}{S}\left(e^{(s \rho)^{2}}+\sigma\right)^{-1}\right|_{0} ^{\infty} \\
& =\frac{\log L^{2}}{4 \pi(1+\sigma)} .
\end{aligned}
$$

The error term is

$$
R=\frac{1}{(2 \pi)^{2}} \int_{\mathbf{R}^{2}} d p\left(p^{\mu}-[p]_{L^{N}}^{\mu}\right) \frac{\partial \widetilde{C}}{\partial p^{\mu}}(\tilde{p}(p))
$$


where $\tilde{p}(p)$ is a point between $p$ and $[p]_{L^{N}}$ and $[p]_{L^{N}}$ denotes the point on the lattice $2 \pi L^{-N} \mathbf{Z}^{2}$ nearest $p \in \mathbf{R}^{2}$. We now note that $\left|p^{\mu}-[p]_{L^{N}}^{\mu}\right|<\mathcal{O}(1) L^{-N}$ and check that

$$
\int d p\left|\frac{\partial \tilde{C}}{\partial p}(\tilde{p}(p))\right|<\mathcal{O}(1) L^{5},
$$

and the result is proved.

\section{References}

[BGN] Benfatto, G., Gallavotti, G., Nicolo, F.: The dipole phase in two-dimensional hierarchical Coulomb gas: Analyticity and correlations decay. Commun. Math. Phys. 106, 227-288 (1986)

[BF] Brydges, D., Federbush, P.: Debye screening. Commun. Math. Phys. 73, 197-246 (1980)

$[B Y]$ Brydges, D., Yau, H.-T.: Grad $\Phi$ perturbations of massless Gaussian fields. Commun. Math. Phys. 129, 351-392 (1990)

[D] Dimock, J.: The Kosterlitz-Thouless phase in a hierarchical model. J. Phys. A23, 1207-1215 (1990)

[DH] Dimock, J., Hurd, T. R.: A renormalization group analysis of QED $_{4}$. preprint (1990)

[FS] Fröhlich, J., Spencer, T.: The Kosterlitz-Thouless transition in two-dimensional Abelian spin systems and the Coulomb gas. Commun. Math. Phys. 81, 527-602 (1981)

[GK] Gawedzki, K., Kupiainen, A.: Block spin renormalization group for dipole gas and $(\nabla \Phi)^{4}$. Ann. Phys. 147, 198-243 (1983), and Lattice dipole gas and $(\nabla \Phi)^{4}$ models at long distances, decay of correlations and scaling limit. Commun. Math. Phys. 92, 531-553 (1984)

[KPW] Kappeler, T., Pinn, K., Wieczerkowski, C.: The renormalization group differential equation and the Kosterlitz-Thouless phase in a hierarchical model, preprint (1990)

[KT] Kosterlitz, J. M., Thouless, D. J.: Ordering, metastability and phase transitions in two-dimensional systems. J. Phys. C6, 1181-1203 (1973)

[MP] Marchetti, D. H. U., Perez, J. F.: A hierarchical model exhibiting the KosterlitzThouless fixed point. Phys. Lett. A118, 74-76 (1986), and The Kosterlitz-Thouless transition in two-dimensional Coulomb gases. J. Stat. Phys. 55, 141-156 (1989)

[MKP] Marchetti, D. H. U., Klein, A., Perez, J. F.: Power law fall off in the Kosterlitz-Thouless phase of a two-dimensional lattice Coulomb gas. J. Stat. Phys. 60, 137

[Y] Yang, W.-S.: J. Stat. Phys. 49, 1-32 (1987)

Communicated by K. Gawedzki 
